\title{
Dynamic Stall on Pitching Cambered Airfoil with Phase Offset Trailing Edge Flap
}

\author{
Farid Samara* and David A. Johnson ${ }^{\dagger}$ \\ University of Waterloo, 200 University Ave W, Waterloo, Canada N2L 3G1
}

\begin{abstract}
Dynamic stall on wind turbine blades often leads to severe fatigue that tends to decrease the lifespan of the blades. To mitigate cyclic loading on the blades, trailing edge flaps (TEF) may be used to control the energy captured by the blades. In this study the influence of a TEF on a pitching S833 cambered airfoil is investigated at a Reynolds number of 170,000 and reduced frequencies of $\boldsymbol{k}=\mathbf{0 . 0 6}$ and 0.1 . The lift and moment hysteresis cycles are presented for mean pitch angles of $0^{\circ}$ and $10^{\circ}$ to represent stall onset and deep stall. The flap, hinged at 0.8 chord, is pitching with different phase lags to study the influence of flap motion. Coefficient of pressure contour plots presented here, clearly indicate the leading edge vortex (LEV) formation and convection. It is concluded that even though the TEF was not capable of controlling the formation of the $\mathrm{LEV}$, it was however capable of reducing the LEV magnitude and more importantly, a reduction in cyclic loading. It is recommended to dynamically pitch the flap out-of-phase with the airfoil motion to reduce the min/max of the lift and moment cycles and reduce negative damping that can lead to stall flutter.
\end{abstract}

\section{Nomenclature}

$$
\begin{array}{ll}
\alpha & =\text { angle of attack, deg } \\
\alpha_{a m p} & =\text { pitching semi-amplitude, deg } \\
\alpha_{F} & =\text { flap angle of attack, deg } \\
\alpha_{\text {mean }} & =\text { pitching mean geometric angle of attack, deg } \\
\alpha_{\text {mean }, F} & =\text { flap pitching mean geometric angle of attack, deg } \\
\mathrm{c} & =\text { chord, m } \\
\mathrm{cw} & =\text { clockwise } \\
\mathrm{ccw} & =\text { counter-clockwise } \\
C_{D} & =\text { coefficient of drag } \\
C_{L} & =\text { coefficient of lift }
\end{array}
$$

\footnotetext{
*Ph.D. Candidate, Wind Energy Group, Mechanical and Mechatronics Engineering; fsamara@ uwaterloo.ca

†Professor, Wind Energy Group, Mechanical and Mechatronics Engineering; david.johnson@uwaterloo.ca
} 


$$
\begin{array}{lll}
C_{M} & =\text { coefficient of moment } \\
C_{p} & =\text { coefficient of pressure } \\
C_{W} & =\text { torsional damping } \\
U_{\infty} & =\text { free stream velocity, } \mathrm{m} \cdot \mathrm{s}^{-1} \\
k & =\text { reduced frequency }\left(k=\pi c f / U_{\infty}\right) \\
f & =\text { frequency of oscillation, } \mathrm{s}^{-1} \\
f_{p} & =\text { pitch frequency of oscillation, } \mathrm{s}^{-1} \\
f_{F} & =\text { flap frequency of oscillation, } \mathrm{s}^{-1} \\
\gamma & =\text { yaw angle, deg } \\
\phi & =\text { phase shift, rad } \\
\rho_{\infty} & =\text { free stream air density, } \mathrm{kg} \cdot \mathrm{m}^{-3} \\
\mathrm{STD} & =\text { standard deviation } \\
\tau & =\text { period of the cycle, } \mathrm{s}
\end{array}
$$

\section{Introduction}

As the demand for more renewable sources of energy grows so does the demand for wind energy generated by horizontal axis wind turbines (HAWT). To make wind turbines more economical and capture more energy, manufacturers are increasing rotor diameter. As the blades get longer the cyclic loading at the root increases causing material fatigue. Cyclic loading is caused by many factors including wind shear, rotor misalignment, and turbulence of the wind resource [1]. Fatigue life governs the design factors for larger wind turbines, so it is important to minimize the cyclic loading [2]. For these reasons, it is imperative to understand and reduce dynamic loading on the blades to increase turbine lifespan and decrease the cost of generated energy. Reduction in blade loading would also decrease the loads on the drivetrain, generator, and tower further reducing the cost of manufacturing and maintenance. Active modification of the blade shape, also known as Smart Rotor, could be used to reduce cyclic loading, material fatigue and unsteady flow conditions [2]. This blade modification would be based on information obtained from different sensors embedded in the wind turbine that measure blade loading.

As wind turbine blades experience unsteady flow conditions the angle of attack $(\alpha)$ on the blade section changes dynamically thus changing the forces experienced by the blades. The $\alpha$ variation could exceed or remain below the static stall angle of the airfoil. There are three airfoil parameters that tend to influence the dynamic stall or unsteady loading: Airfoil shape, mean pitching angle/amplitude, and the reduced frequency $k=\pi c f / U_{\infty}$ ( $c$ is the chord, $f$ is the frequency and $U_{\infty}$ is the freestream velocity) [3, 4]. Corke and Thomas [5] defined the flow field to be unsteady when $k$ is higher than 0.05 otherwise unsteady effects can be neglected. They also explained the reduced frequency by its 
analogy to a phase lag parameter where the fluid reaction lags behind the pitching motion due to inertial effects.

Dynamic stall is characterized by extreme hysteresis and non-linear cycles in the coefficient of lift $\left(\mathrm{C}_{\mathrm{L}}\right)$, coefficient of moment $\left(\mathrm{C}_{\mathrm{M}}\right)$ and coefficient of drag $\left(\mathrm{C}_{\mathrm{D}}\right)$ [4]. This causes the aerodynamic forces acting on the blade to be higher than their static counterparts. The forces on the blades are not just higher but dynamic stall generates periodic and oscillatory motion that tends to increase the stress and fatigue levels at the root of the blade. The dynamic stall cycle could be split into four sections: pitch-up motion, flow separation, pitch-down motion, and flow re-attachment. During pitch-up motion the flow remains attached to the airfoil thus $\mathrm{C}_{\mathrm{L}}$ keeps increasing past the static stall angle. This is due to the pitching motion and the formation of a leading edge vortex (LEV) or dynamic stall vortex [5]. The LEV brings additional suction to the leading edge and produces values of $\mathrm{C}_{\mathrm{L}}, \mathrm{C}_{\mathrm{M}}$ and $\mathrm{C}_{\mathrm{D}}$ that are much higher than their static counterparts during pitch-up motion [4]. As the airfoil starts to pitch down, the LEV convects downstream over the suction side and once the LEV flows past the trailing edge the flow separates leading to a severe loss in lift, increase in nose-down pitching moment and increase in drag. In some cases a secondary LEV is formed and shed, leading to another $\mathrm{C}_{\mathrm{L}}$ peak [6]. Gharali and Johnson [7.-9] reported the formation of a secondary LEV and in some cases, smaller vortices after that. The secondary LEV tends to be weaker in magnitude when compared to the first LEV. Lee and Gerontakos [10] have also reported the formation of a secondary LEV. Numerical investigations were able to simulate the formation of both the first and secondary LEV [11]. Typically, during pitch-down, the airfoil is stalled even though $\alpha$ is below the static stall angle. The flow then re-attaches to the surface as $\alpha$ decreases and the cycle starts again [10, 12, 14]. Leishman[6] and recently Masdari et al.[15] studied the effects of the mean angle of attack, pitching amplitude, and reduced frequency on dynamic stall.

While the airfoil is pitching close to the stall point, aeroelastic stability problems could arise. Aeroelastic divergence or stall flutter is promoted when the torsional damping (defined by the line integral $C_{W}=\oint C_{m}(\alpha) d \alpha$ ) is negative [16]. Damping is positive when it corresponds a counter-clockwise (ccw) loop while negative for a clockwise (cw) loop in the $\mathrm{C}_{\mathrm{M}}-\alpha$ plot [5]. During negative damping, vibration can increase significantly and may lead to structural damage if the structural dynamics permit [17]. Using a TEF to mitigate negative damping without significant loss of lift would benefit the turbine system greatly and would ensure limited flutter on the blades [17]. Davis et al. [18] used CFD simulation to show that TEF are capable of reducing negative damping and flutter on rotor blades.

Surface pressure measurements have been used in the past to determine the magnitude and development of dynamic stall and the LEV by Leishman [6], McCroskey et al. [19] and others. These studies show how the LEV could be identified using surface pressure measurements alone. Surface pressure could also be used to determine the location of the laminar-turbulent transition point and the laminar separation bubble. The transition point is associated with high pressure fluctuations that could be measured through surface pressure. Boutilier and Yarusevych [20] showed that the maximum root-mean-square (RMS) in surface pressure corresponds to a location between the mean transition and reattachment point. Raiola et al. [21] was able to determine the transition from the standard deviation (STD) in $\mathrm{C}_{\mathrm{p}}$ 
contour plots while the airfoil was pitching.

Dynamic stall produces load fluctuations that are cyclic and repetitive in nature. The cyclic loading experienced by wind turbine blades could be characterized by the min/max load value difference and the hysteresis in the cycle. The main aim of the TEF is to reduce the difference between the $\min / \mathrm{max}$ load cycle and reduce the hysteresis cycle during wind turbine operation while ideally eliminating the negative damping. Ideally, if the cyclic loading were eliminated, the load magnitude on the blade would be invariant. To decrease the cyclic loading caused by dynamic stall it is important to control the development of the LEV and eliminate or decrease the detrimental hysteresis lift and moment cycle. Different control strategies have been developed previously such as leading edge blowing or suction [22, 23], synthetic jets [24], leading edge plasma actuation [25, 26], vortex generators [27, 28] and trailing edge flaps (TEF) [29, 32]. Barlas and van Kuik [2] summarized and reviewed the different control strategies mentioned to reduce fatigue loading on wind turbine blades. TEF were found to be the most efficient of the control strategies tested because of their control authority over the coefficients of lift and drag, linearity, high frequency response and their simplicity of use. In comparison, they found microtabs are less efficient for detailed load control due to their on-off characteristics; camber control is expensive, complex to design, and has high power consumption; boundary layer strategies are limited by their control authority.

Lee et al. [33-36] produced several papers investigating how a TEF can influence the formation and detachment of LEV. Green et al. [17] discuss the benefit of TEF and how it influences dynamic stall behavior. It was reported that positive flap deflection, defined in this paper as deflection towards the suction side (See Figure 3), was capable of reducing the hysteresis cycle in the lift and moment curve and negative damping possibly due to stall vortex deflection or a weaker and thinner LEV. The TEF was not however capable of controlling the formation and detachment of the LEV but the TEF was capable of controlling the LEV magnitude. Raiola et al. [21] showed that a TEF on a NACA0015 airfoil was capable of controlling the loads generated by dynamic stall and the LEV. The literature lacks data about dynamic stall behavior on wind turbine specific airfoils or cambered airfoils in general with surface pressure measurements.

The literature review presented discusses dynamic stall on a symmetric airfoil such as the NACA 0012 or NACA 0015. More research is aimed at rotorcraft engineering and less work has been solely aimed at wind turbine blades. This paper builds on prior studies and specifically discusses the S833 cambered airfoil by NREL that is designed for small scale wind turbines [37]. To do so, an experimental campaign was initiated to study TEF on a wind turbine beginning with characterizing a 2D turbine blade element in a closed loop wind tunnel at a chord $R e=170,000$ and reduced frequencies $k=0.06$ and 0.1 . Time resolved surface pressure measurements on the airfoil element have been obtained for different dynamic cases and flap motion. A few of the novel ideas introduced in this paper: the design and actuation of a TEF in a small section airfoil, simultaneous surface pressure measurements where the transducers are embedded in the airfoil, and dynamic stall studies on a wind turbine specific airfoil with a flapping trailing edge. 


\section{Experimental Setup}

First the instrumented airfoil model will be explained briefly along with the wind tunnel setup. Secondly, the airfoil motion and different test cases will be presented. Finally the validation for the differential pressure measurement will be discussed.

\section{A. Wind Tunnel Setup}

The experimental campaign was carried out in the closed loop wind tunnel at the University of Waterloo. The wind tunnel has a contraction ratio of 9:1 and a cross section of $0.61 \mathrm{~m}$ square. The uniformity was found to be within \pm $0.4 \%$ in the spanwise and vertical direction. The turbulence intensity was $0.1 \%$. The free-stream velocity was set by the static pressure drop across the contraction with an uncertainty of less than $2.5 \%$. The blockage ratio was around $8 \%$. The wind speed of operation was set to $15 \mathrm{~m} / \mathrm{s}$ resulting in a chord-based Reynolds number $(\operatorname{Re})$ of 170,000 . This velocity was based upon a compromise between high Reynolds number and an achievable pitch frequency to achieve dynamic stall behavior on the airfoil. More information about the wind tunnel calibration could be found in [38].

The NREL S833 airfoil [37], designed for small wind turbines, was used for the experiments. The flap width was chosen to be $20 \%$ of the $178 \mathrm{~mm}$ chord while the flap span was $60 \%$ of the $584 \mathrm{~mm}$ airfoil span. To incorporate the sensors and actuators the aerodynamic blade was 3D printed using ABS-M30 material in three different parts: the suction side, pressure side, and the flap. Those three sections were printed and polished to achieve a smooth surface finish. A stainless steel spar at the quarter chord was used to support the 3D printed blades and hold all the components together. The airfoil was then cantilevered and supported on the drive side in the wind tunnel. A schematic of the experimental setup is shown in Figure 1. The designed model was capable of housing all the instrumentation and the flap actuation system which consists of a Maxon EPOS 24/2 controller and a drive assembly. The flap drive assembly has a diameter of $8 \mathrm{~mm}$ and length of $40 \mathrm{~mm}$ and consists of a motor, a 256:1 gearbox and a quadrature encoder with 4096 counts per turn. The backlash in the system at the gearbox output side is $2.5^{\circ}$. The motor and controller were placed inside the airfoil and the motor was directly coupled to the flap shaft. The setup of the flap motion control could be seen in Figure 2 .

To determine the coefficient of lift and moment, 54 surface pressure taps were located on an aluminum airfoil section at midspan with 4 of those pressure taps located on the flap. The pressure taps were laid out in a zigzag pattern to reduce unwanted turbulence interference effects from the upstream taps. The distance between the two planes of pressure taps is $8 \mathrm{~mm}$ or $1.4 \%$ of the airfoil span. The aluminum pressure tap section was placed at the center of the wind tunnel section sandwiched between the 3D printed airfoil pieces. The internal diameter of the taps is 0.4 mm while the depth of the taps is $2 \mathrm{~mm}$. Tubing with an ID of $1.6 \mathrm{~mm}$ was used to connect the taps to the transducers. The maximum length of the tubing was $15 \mathrm{~cm}$ but most of the lengths were under $5 \mathrm{~cm}$. Based on the tube length and tap dimensions and using the Helmholtz resonator model [39], the frequency response of the surface pressure measurements is $15 \mathrm{kHz}$. To measure 
the surface pressure on the airfoil at the frequency desired, pressure measurements had to be simultaneous. To achieve this, 27 differential pressure transducers were used to measure the differential pressure between the suction and pressure side of the airfoil at the same x/c location. This technique was successfully used by Raiola et al. [21] on a symmetric airfoil. To validate the technique used on cambered airfoils, a set of experiments were conducted and are discussed in Section II.C. The miniature pressure transducers (All Sensors model $120 \mathrm{cmH} 2 \mathrm{O}-\mathrm{D} 1-4 \mathrm{~V}-\mathrm{MINI}$ ) were used in this setup with a pressure range of $-0.1 \mathrm{kPa}$ to $11.8 \mathrm{kPa}$. This high range was chosen because after the $2 \mathrm{D}$ wind tunnel experiments, the same rig is being used on a full scale turbine operating under different conditions including a much higher Reynolds number. The uncertainty error due to linearity and hysteresis is $\pm 0.05 \%$ of full scale. The calculated uncertainty due to the pressure transducers and the data acquisition system (DAQ) was $\pm 51 \mathrm{~Pa}$. The $3 \mathrm{D}$ printed airfoil model was capable of housing the pressure transducers and all the tubing needed to connect the transducers to the pressure taps. Refer to Figure 2 for more clarification. The coefficient of pressure $\left(\Delta \mathrm{C}_{\mathrm{p}}\right)$ was calculated based on the following equation $\Delta \mathrm{C}_{\mathrm{p}}=\left(p_{\text {suction side }}-p_{\text {pressure side }}\right) /\left(\frac{1}{2} \rho_{\infty} U_{\infty}^{2}\right)$ where $\rho_{\infty}$ is the free stream fluid density. Finally, to measure the signal from the transducers, the NI 9205 DAQ card by National Instruments was used which has 32 channels. The DAQ card measured the signal sequentially and the time it took to measure all 28 signals was 0.11 millisecond or in about $0.02^{\circ}$ of airfoil motion. For this reason, the data acquisition was considered virtually simultaneous. More details about the experimental setup could be found in Samara and Johnson [40].

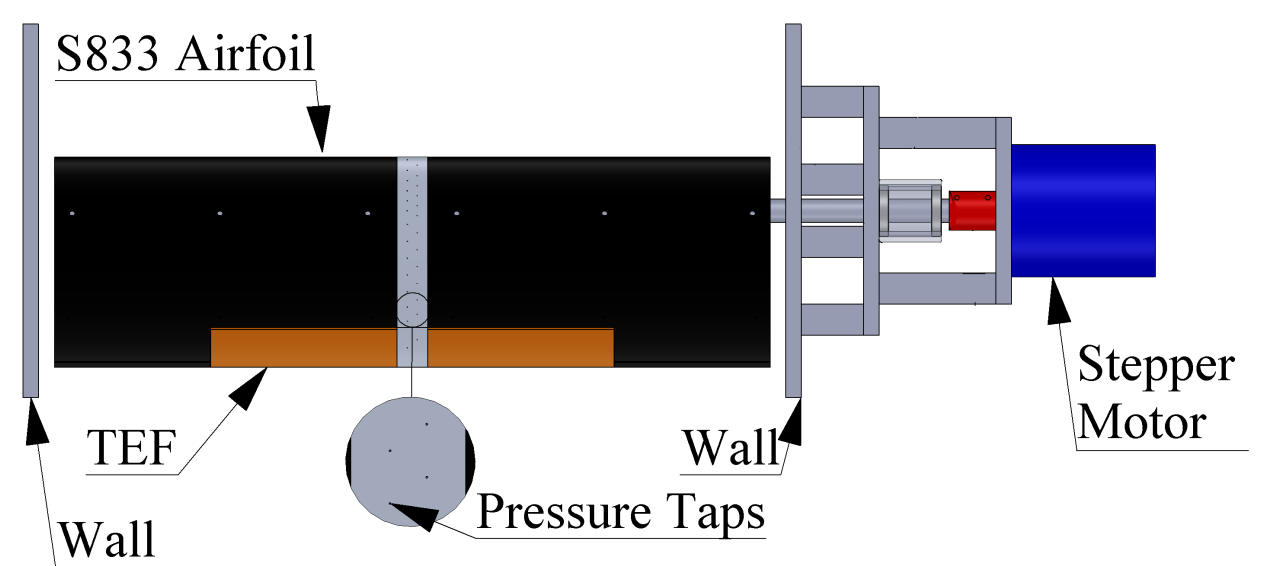

Fig. 1 A schematic showing how the airfoil was setup inside the wind tunnel for characterization.

\section{B. Airfoil Motion}

On a rotating wind turbine blade the effective angle of attack $(\alpha)$ varies as the blade rotates for a yawed turbine. Morote [41] and Burton et al. [42] modeled the $\alpha$ variation as a function of azimuthal position. Gallant and Johnson [43] compared those previous two models to experimental data on a 3.4 m diameter HAWT. From the models and experiments it was found that the $\alpha$ variation is very similar to a sinusoidal path when the turbine is yawed. To simulate 


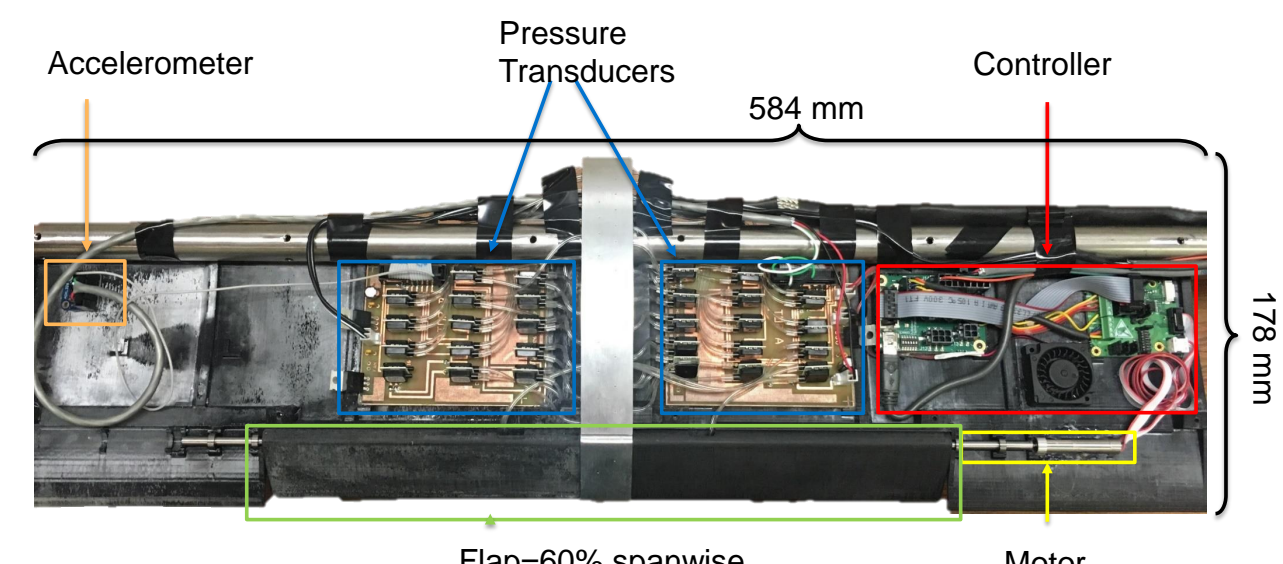

Flap $=60 \%$ spanwise

Motor

Fig. 2 Internal organization of smart rotor blade with cover removed.

the dynamic forces on a yawed wind turbine blade, $\alpha$ will follow a sine path as described in Gharali and Johnson [9] and described in equation $1(\alpha(t))$. To study the influence that the TEF has on the aerodynamic forces on the airfoil, the flap angle oscillation frequency, $f_{F}$, is the same as the pitch frequency $f_{p}$. Equation 2 describes the motion for the flap angle $\alpha_{F}(\mathrm{t})$.

$$
\begin{gathered}
\alpha(t)=\alpha_{\text {mean }}+\alpha_{a m p} \sin \left(2 \pi f_{p} t\right) \\
\alpha_{F}(t)=\alpha_{a m p, F} \sin \left(2 \pi f_{F} t+\phi\right)
\end{gathered}
$$

where $f_{p}$ is the frequency of airfoil oscillation, $\alpha_{a m p}$ is the semi-amplitude for the pitch, $\alpha_{m e a n}$ is the mean geometric angle of attack of the airfoil, $\alpha_{a m p, F}$ is the semi-amplitude of flap angle and $\phi$ is the phase shift with respect to the pitch motion. Figure 3 shows the positive direction for the pitch and flap angles. The system is operated at two different reduced frequencies, $k$, being 0.06 and 0.1 to represent the $\alpha$ variation at two different spanwise locations on a yawed wind turbine blade. The wind turbine blade element section discussed will subsequently be installed on a wind turbine with a $3.6 \mathrm{~m}$ diameter and a rotational speed of $200 \mathrm{rpm}$. On the turbine, $k$ will decrease when the blade element is moved away from the hub. A $k$ value of 0.06 would occur closer to the blade tip while $k$ of 0.1 would occur closer to the hub. The different test cases reported here are shown in Table 1 Test case $a$ and $b$ represent a reduced frequency of 0.06 and 0.1 respectively while test case 1 and 2 represent $\alpha_{\text {mean }}=0^{\circ}$ and $10^{\circ}$ respectively. $\alpha_{\text {mean }}=0^{\circ}$ is chosen because $\alpha$ will not exceed the static stall angle, while $\alpha_{\text {mean }}=10^{\circ}$ pushes $\alpha$ beyond static stall to promote dynamic stall. Four different flap phases $\phi=0 \pi, \phi=\pi / 2, \phi=\pi$, and $\phi=3 \pi / 2$ will be tested.

To better understand the motion of the airfoil and flap with different flap phases, Figure 4 graphically depicts the kinematic motion along with airfoil/flap positions for case 1a or $1 \mathrm{~b}$ where $\alpha_{\text {mean }}=0^{\circ}$. The four different flap phases 


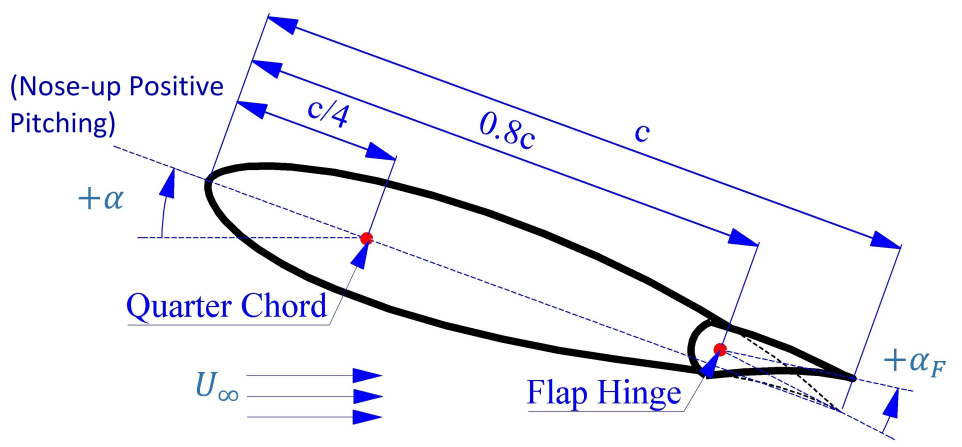

Fig. 3 A sketch illustrating the airfoil and flap degrees of freedom. $+\alpha$ positive angle of attack; $+\alpha_{F}$ positive flap angle; $U_{\infty}$ freestream velocity

Table 1 Wind tunnel dynamic pitching test cases

\begin{tabular}{cccccccc}
\hline \hline Test No & $\alpha_{\text {mean }}\left({ }^{\circ}\right)$ & $f_{p}(\mathrm{~Hz})$ & $k$ & $f_{F}(\mathrm{~Hz})$ & $\alpha_{a m p}\left({ }^{\circ}\right)$ & $\alpha_{a m p, F}\left(^{\circ}\right)$ & $\phi$ \\
\hline \hline Case 1a & 0 & 1.61 & 0.06 & {$[0,1.61]$} & 10 & 20 & {$[0: 0.5: 1.5] \pi$} \\
\hline Case 1b & 0 & 2.68 & 0.1 & {$[0,2.68]$} & 10 & 20 & {$[0: 0.5: 1.5] \pi$} \\
\hline Case 2a & 10 & 1.61 & 0.06 & {$[0,1.61]$} & 10 & 20 & {$[0: 0.5: 1.5] \pi$} \\
\hline Case 2b & 10 & 2.68 & 0.1 & {$[0,2.68]$} & 10 & 20 & {$[0: 0.5: 1.5] \pi$} \\
\hline \hline
\end{tabular}

are plotted along with the airfoil motion schematics at four instances in the cycle. The arrows on the airfoil motion schematic show the direction of motion of the airfoil and flap.

\section{Differential Pressure Measurement Validation}

In order to validate the approach of using a differential pressure measurement to determine $\mathrm{C}_{\mathrm{L}}$ and $\mathrm{C}_{\mathrm{M}}$ on a cambered airfoil, independent single ended pressure measurements of the suction and pressure sides were compared with the differential measurements. The tubing inside the airfoil was modified to conduct single ended pressure measurement and then modified again to conduct differential pressure measurements. The validation was done for case $2 \mathrm{~b}$ where $k=0.1$ and $\alpha_{\text {mean }}=10^{\circ}$. This case was chosen because of its higher $\alpha_{\text {mean }}$ and high reduced frequency that produced the highest cyclic loading.

Figure 5 compares the $\mathrm{C}_{\mathrm{L}}$ and $\mathrm{C}_{\mathrm{M}}$ cycle while the airfoil is operating in the dynamic stall regime using two approaches: single ended surface pressure measurements and differential surface pressure measurements. The difference between the two cases is very small in magnitude while the patterns are exactly the same. The difference in $\mathrm{C}_{\mathrm{L}}($ Figure 5a between the two cases is negligible for lower $\alpha$ but increases for higher $\alpha$. This is because, in the differential pressure measurement, the pressure force from each tap angle is projected to the global y-coordinate using an average angle of the suction and pressure tap. On the other hand, single ended pressure measurements use each tap angle to project the pressure force to the global y-coordinate producing the most accurate results. As for the $\mathrm{C}_{\mathrm{M}}$ (Figure $5 \mathrm{~b}$ ) calculations, there was no need to project the tap angle to the global y-coordinate. For this reason the $\mathrm{C}_{\mathrm{M}}$ values in both cases are very similar and independent of $\alpha$. The maximum difference is $4 \%$ for $\mathrm{C}_{\mathrm{L}}$ and less than $1 \%$ for $\mathrm{C}_{\mathrm{M}}$. The difference in 

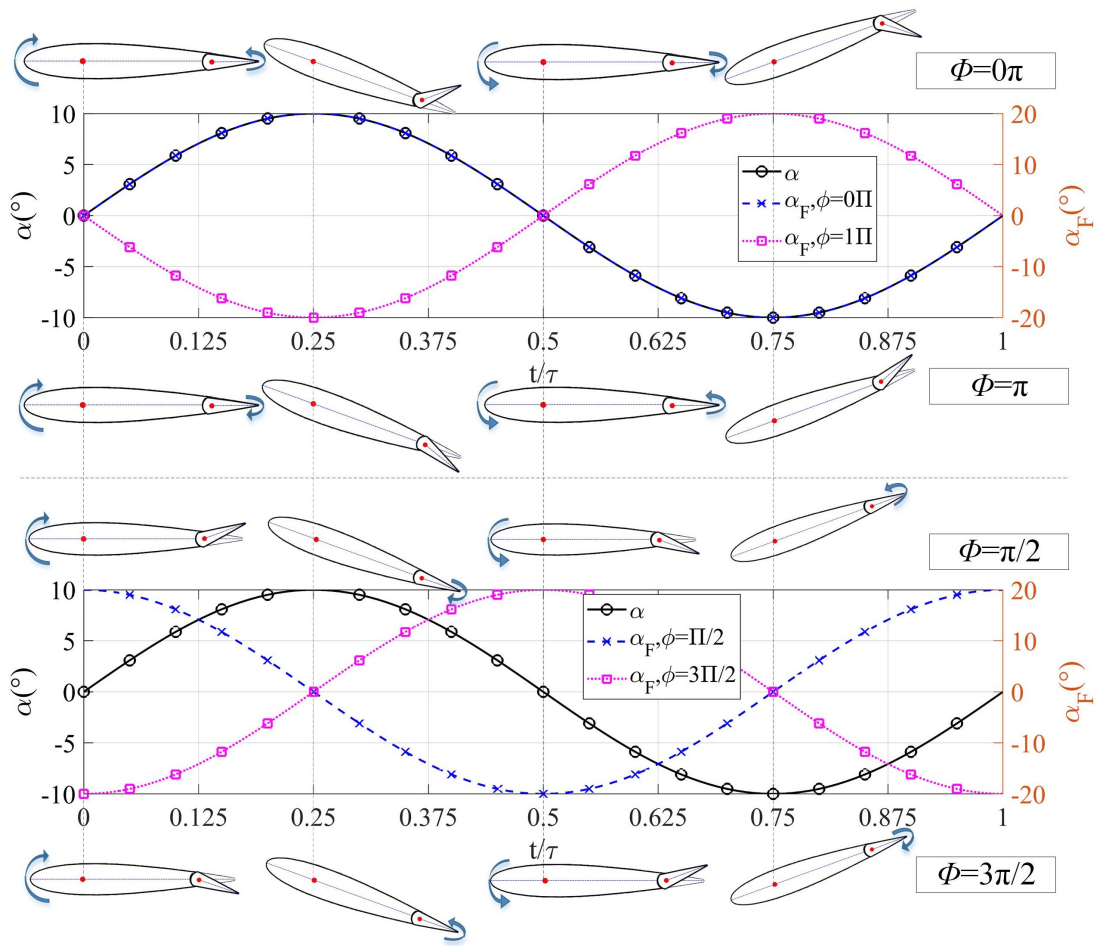

Fig. 4 Kinematics of the airfoil and TEF for $\alpha_{\text {mean }}=0^{\circ}$ and different $\phi$ for case 1a or $1 \mathrm{~b}$

$\Delta \mathrm{C}_{\mathrm{p}}$ (that produced both coefficients) between the two cases was also studied and the largest difference was found to be concentrated at the LE and when $\alpha=20^{\circ}$. This is because the curvature of the airfoil is most significant at the LE and $\alpha$ is high. These differences are minor and do not significantly change the details including how the LEV is interpreted and studied. Since this study focuses on how the TEF influences surface pressure and the development of the LEV, these constant errors and offsets will be present in all cases and will not affect the comparison. In conclusion, the two methods are both valid because the difference with the two cases is small and could be ignored and because the pattern in $\mathrm{C}_{\mathrm{L}}$ and $\mathrm{C}_{\mathrm{M}}$ is not influenced by differential pressure measurement. 


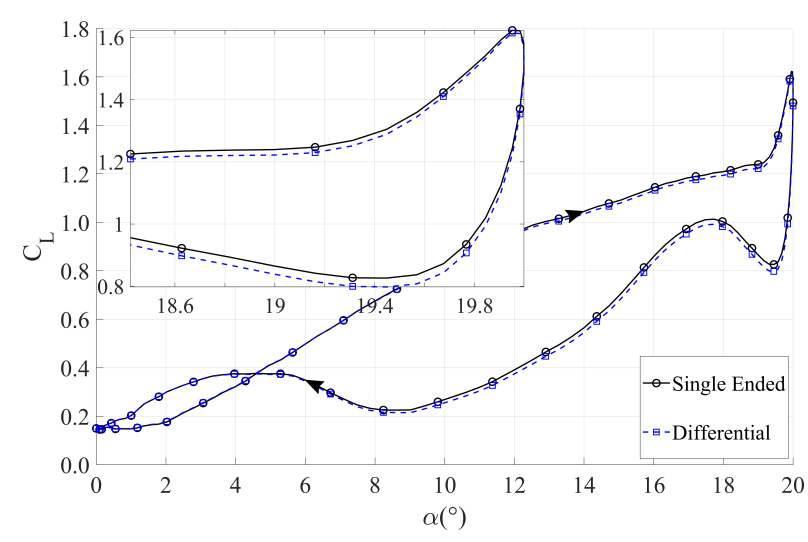

(a) $\mathrm{C}_{\mathrm{L}}$ versus $\alpha$ for both pressure measurement techniques

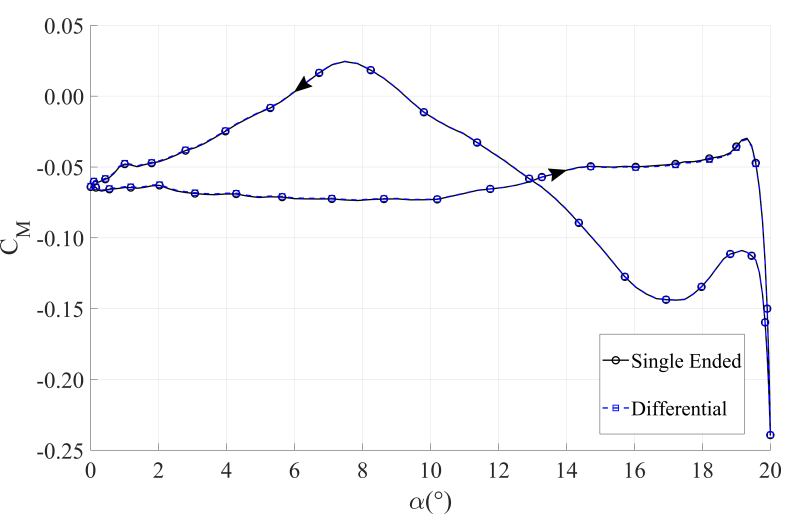

(b) $\mathrm{C}_{\mathrm{M}}$ versus $\alpha$ for both pressure measurement techniques

Fig. 5 Coefficient of a) lift $\left(\mathrm{C}_{\mathrm{L}}\right)$ and $\left.\mathrm{b}\right)$ moment $\left(\mathrm{C}_{\mathrm{M}}\right)$ versus $\alpha$ for case $2 \mathrm{~b}\left(k=0.1\right.$ and $\left.\alpha_{\text {mean }}=10^{\circ}\right)$ to compare single ended pressure measurements to differential pressure measurements. A plot inset is used to magnify the data around $\alpha=19^{\circ}$ for $\mathrm{C}_{\mathrm{L}}$.

\section{Results}

Now that the experimental setup and validation has been introduced, the results for the experimental campaign are presented. The static airfoil characterization is discussed briefly for different flap angles so it could be presented as a comparison for the dynamic cases. The dynamic results will then be presented for $\alpha_{\text {mean }}=0^{\circ}$ and $\alpha_{\text {mean }}=10^{\circ}$.

\section{A. Static Airfoil Characterization}

Figures $6 \mathrm{a}$ and $6 \mathrm{~b}$ show the lift coefficient $\left(\mathrm{C}_{\mathrm{L}}\right)$ and moment coefficient $\left(\mathrm{C}_{\mathrm{M}}\right)$ at the quarter chord versus $\alpha$, respectively. $\mathrm{C}_{\mathrm{M}}$ is defined as positive for nose-up pitching moment and negative for nose-down pitching moment. The measured angle of attack $(\alpha)$ ranged from $-5^{\circ}$ to $50^{\circ}$ in steps of $1^{\circ}$ and the flap angle $\left(\alpha_{F}\right)$ from $-20^{\circ}$ to $20^{\circ}$ in steps of $10^{\circ}$. This wide range of $\alpha$ was chosen to replicate the same range that wind turbine blades experience [43, 44]. Both coefficients are determined by integrating the differential coefficient of pressure $\left(\Delta\left(\mathrm{C}_{\mathrm{p}}\right)\right)$ along the chord of the airfoil from the 27 differential pressure transducers. Corrections due to wind tunnel blockage and sidewalls were calculated to be around $4 \%$ and $2 \%$ for $\mathrm{C}_{\mathrm{L}}$ and $\mathrm{C}_{\mathrm{M}}$ respectively [45, 46]. Results from XFoil [47] with $\alpha$ ranging from $-3^{\circ}$ to $20^{\circ}$ for a clean airfoil $\left(\mathrm{N}_{\text {crit }}=9\right)$ with no flap at the same Re are also plotted on both figures for comparison. In Figure 6a the experimental values for $\alpha_{F}=0^{\circ}$ match the XFoil results for $\alpha<5^{\circ}$. The measurements and the model start to deviate slightly when $\alpha$ is higher than $5^{\circ}$ with XFOIL predicting higher values. This is possibly due to the discretization of the $\Delta\left(\mathrm{C}_{\mathrm{p}}\right)$ measurements obtained from the pressure taps along the chord, the flap/airfoil intersection line on the physical model or incorrect assumptions in XFoil. Although the magnitude does not match, the trend in the data is similar near the stall point and at zero lift. Experimental $\mathrm{C}_{\mathrm{L}}$ and $\mathrm{C}_{\mathrm{M}}$ measurement data of a $\mathrm{S} 809$ airfoil from Ramsay et al. [46] are plotted in Figure 6 The data for S809 matches well with S833 for $\alpha<10^{\circ}$, but diverges for higher $\alpha$. This occurs because Re for the $\mathrm{S} 809$ is 750,000 while Re for the S833 is 170,000. Reported experimental data for the S833 airfoil 
could not be found in the literature. Data from Ramsay et al. on the S809 airfoil is presented as it is the closest match because the airfoils are somewhat similar and data is provided for high $\alpha$. This data is shown, to compare the dynamics of stall and how $\mathrm{C}_{\mathrm{L}}$ increases after $\alpha=20^{\circ}$ when the airfoil is stalled.

As the flap angle, $\alpha_{F}$, decreases (increasing the camber of the airfoil), $\mathrm{C}_{\mathrm{L}}$ increases while $\mathrm{C}_{\mathrm{M}}$ decreases. The opposite is true for increasing the flap angle and decreasing airfoil camber. For example at $\alpha_{F}=-20^{\circ} \mathrm{C}_{\mathrm{L}}$ increased by $23 \%$ while for $\alpha_{F}=+20^{\circ} \mathrm{C}_{\mathrm{L}}$ decreased by $28 \%$ based on $\alpha_{F}=0^{\circ}$ and $\alpha=11^{\circ}$. The average representative uncertainty in $\mathrm{C}_{\mathrm{L}}$ and $\mathrm{C}_{\mathrm{M}}$ was calculated to be \pm 0.069 and \pm 0.014 respectively. The uncertainty was based primarily on the error in the pressure transducers and wind tunnel velocity determination. The uncertainty in $\alpha$ and $\alpha_{F}$ is $\pm 0.1^{\circ}$ and $\pm 2^{\circ}$ respectively.

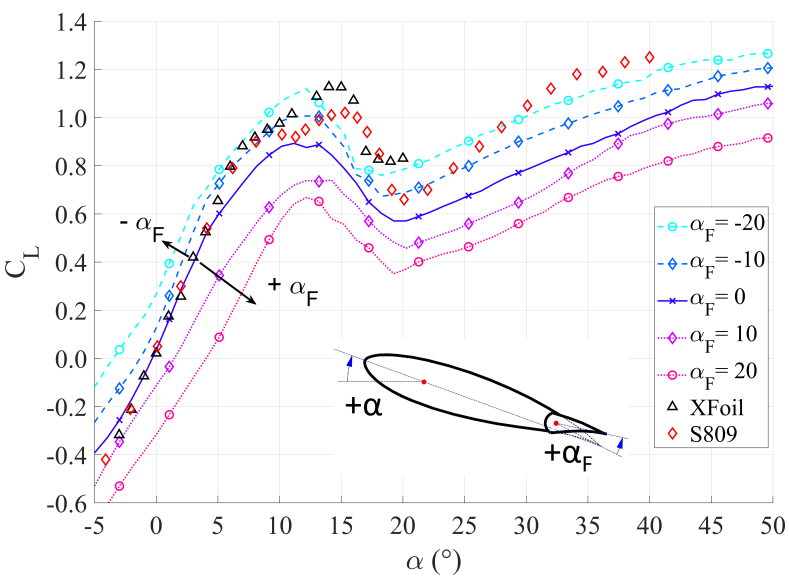

(a) Coefficient of lift

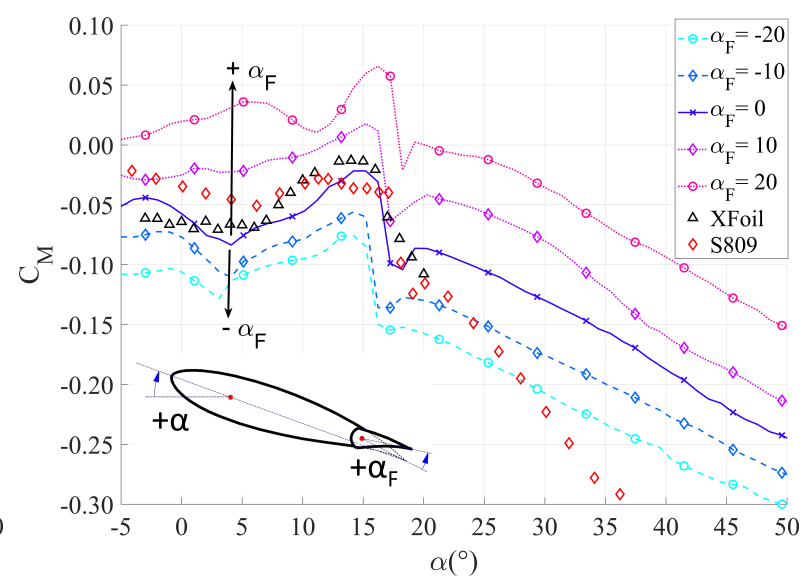

(b) Coefficient of moment

Fig. 6 Measured coefficient of a) lift $\left(\mathrm{C}_{\mathrm{L}}\right)$ and b) moment $\left(\mathrm{C}_{\mathrm{M}}\right)$ versus $\alpha$ for several $\alpha_{F}$. Only every fourth data point is marked on the plot for clarity. The experimental data is compared to XFoil [47] predictions for a clean airfoil $\left(\alpha_{F}=0\right)$. Experimental $\mathrm{C}_{\mathrm{L}}$ and $\mathrm{C}_{\mathrm{M}}$ for the $\mathrm{S} 809$ airfoil at a higher Re are also plotted from reference [46].

Contour plots of $\Delta \mathrm{C}_{\mathrm{p}}$ for $\mathrm{x} / \mathrm{c}$ locations versus $\alpha$ at $\alpha_{F}$ of $-20^{\circ}$ and $20^{\circ}$ are shown in Figures $7 \mathrm{p}$ and $7 \mathrm{p}$. The contour plots are a matrix of 27 averaged pressure points in the x-direction by 55 different $\alpha$ angles in the y-direction with each point being an average of 4000 data points. For some instances of $\alpha_{F}$ and $\alpha$ angles, $\Delta \mathrm{C}_{\mathrm{p}}$ is plotted in Figure 7 . The contour plots clearly show the stall angle occurring when the $-\Delta \mathrm{C}_{\mathrm{p}}$ peak close to the $\mathrm{LE}(0<\mathrm{x} / \mathrm{c}<0.1)$ is sharply reduced. From Figures 6 and 7 the stall angle is $16^{\circ}, 17^{\circ}$ and $18^{\circ}$ for $\alpha_{F}=-20^{\circ}, \alpha_{F}=0^{\circ}$ and $\alpha_{F}=20^{\circ}$ respectively. The flap angle, $\alpha_{F}$, thus has influence over the stall angle of the airfoil as the camber of the airfoil changes. The change in $\alpha_{F}$ increases and decreases the $-\Delta \mathrm{C}_{\mathrm{p}}$ peak magnitude in pre-stall conditions thus increasing and decreasing $\mathrm{C}_{\mathrm{L}}$. Changing $\alpha_{F}$ from $-20^{\circ}$ to $20^{\circ}$ changes the sign of $-\Delta \mathrm{C}_{\mathrm{p}}$ values close to the TE from positive to negative. This indicates that the suction side of the airfoil has both negative and positive pressure that causes pressure imbalance on the airfoil that leads to much larger $\mathrm{C}_{\mathrm{M}}$ values. Just after the stall point $\left(\alpha=17^{\circ}\right)$, the $-\Delta \mathrm{C}_{\mathrm{p}}$ peak close to the $\mathrm{LE}(0<\mathrm{x} / \mathrm{c}<0.1)$ is dramatically reduced but $-\Delta \mathrm{C}_{\mathrm{p}}$ over the entire chord is slightly increased (this is more clearly seen in Figure $7 \mathrm{~F}$ ). This 
indicates a movement of the pressure center from the LE to the mid-chord region. The pressure center movement leads to a dramatic change in $\mathrm{C}_{\mathrm{M}}$ that was also seen in Figure $6 \mathrm{~b}$. Uncertainty in $\Delta \mathrm{C}_{\mathrm{p}}$ is calculated to be around 0.085.

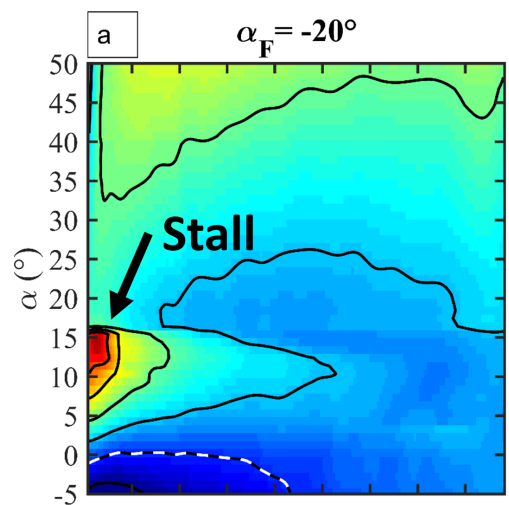

$\begin{array}{llllllllll}0.1 & 0.2 & 0.3 & 0.4 & 0.5 & 0.6 & 0.7 & 0.8\end{array}$

$\mathrm{x} / \mathrm{c}$

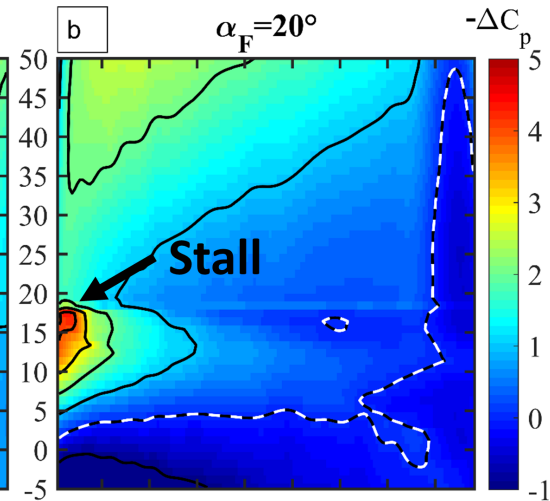

$\begin{array}{lllllllll}0.1 & 0.2 & 0.3 & 0.4 & 0.5 & 0.6 & 0.7 & 0.8\end{array}$ $\mathrm{x} / \mathrm{c}$

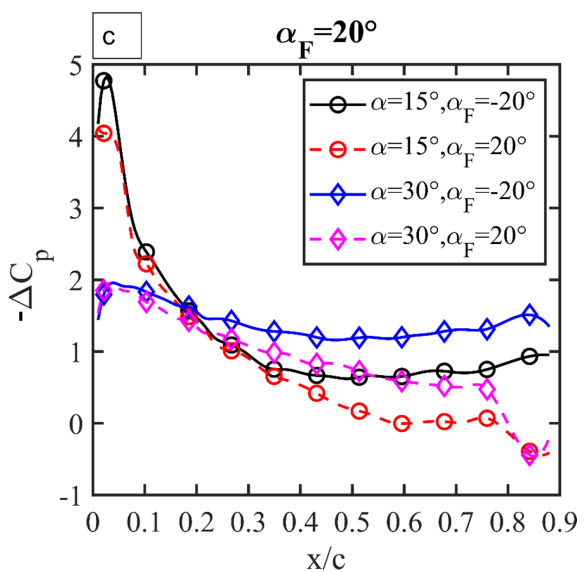

Fig. 7 Contours of $-\Delta \mathrm{C}_{\mathrm{p}}$ versus $\mathrm{x} / \mathrm{c}$ versus $\alpha$. a) $\alpha_{F}=-20^{\circ}$ and $\left.\mathbf{b}\right) \alpha_{F}=20^{\circ}$. The location of stall is marked for clarity. The

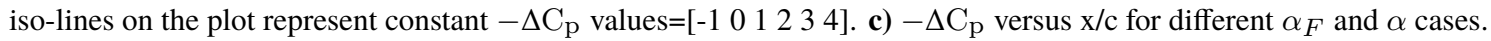

\section{B. Dynamic Pitching}

In this section, the dynamic pitching results will be presented. The motion of the airfoil and flap are governed by Equations 1 and 2 while the study parameters are presented in Table 1 . Results for $\alpha_{m e a n}=0^{\circ}$ and $10^{\circ}$ are presented separately in the following two sections.

1. $\alpha_{\text {mean }}=0^{\circ}$

The Figures presented in this section belong to cases $1 \mathrm{a}$ and $1 \mathrm{~b}$ where $k=0.06$ and 0.1 respectively with $\alpha_{m e a n}=0^{\circ}$. Approximately 600 cycles were collected for each scenario and each cycle was binned into 160 bins based on cycle period. $\mathrm{C}_{\mathrm{L}}$ and $\mathrm{C}_{\mathrm{M}}$ were then calculated for each of the 160 bins in the cycle. The $\mathrm{C}_{\mathrm{L}}$ averaged cycles are presented in two plots, Figures $8 \mathrm{a}$ and $8 \mathrm{c}$, and each plot contains a FixedFlap loop, static airfoil data, and 2 flap phases (First: $\phi=0 \pi$ and $\pi / 2$, second: $\phi=\pi$ and $3 \pi / 2$ ). The arrows on the plot indicate the direction of airfoil motion, whether it would be upstroke or downstroke. The FixedFlap data represents a pitching clean airfoil where the flap is not moving relative to the airfoil. The four phases shown represent the phase shift between the pitch and the flap motion denoted by $\phi$ in Equation 2. The $\mathrm{C}_{\mathrm{M}}$ averaged cycles are also presented in a similar fashion in Figures $8 \mathrm{~b}$ and $8 \mathrm{~d}$.

Looking at Figures $8 \mathrm{a}$ and $8 \mathrm{c}$ for case 1a, the dynamic FixedFlap results could be compared to the measured static airfoil $\mathrm{C}_{\mathrm{L}}$ marked in red stars $(\star)$. The hysteresis cycle due to dynamic motion produces higher $\mathrm{C}_{\mathrm{L}}$ values in comparison to the static case as expected. The largest change in $\mathrm{C}_{\mathrm{L}}$ for $\phi=0 \pi$ or $\pi$, when compared to the FixedFlap case, is found to be when $\alpha$ is at its $\min \left(-10^{\circ}\right)$ and $\max \left(10^{\circ}\right)$. When $\phi=0 \pi$ or $\pi$ and $\alpha=0^{\circ}$ then $\alpha_{F}=0^{\circ}$ producing similar conditions to the FixedFlap case thus making all three curves align at that $\alpha$ value. When $\phi=0 \pi$ and $\alpha=10^{\circ}$ 


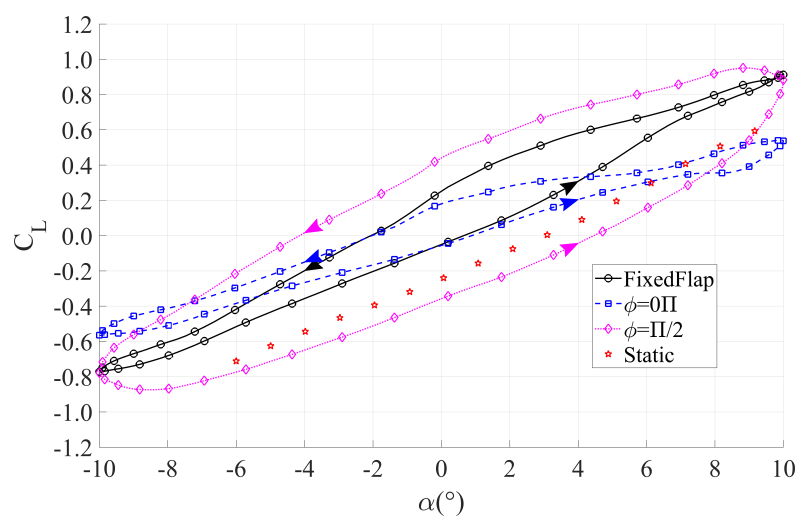

(a) Coefficient of lift $(\phi=0 \pi, \phi=\pi / 2)$

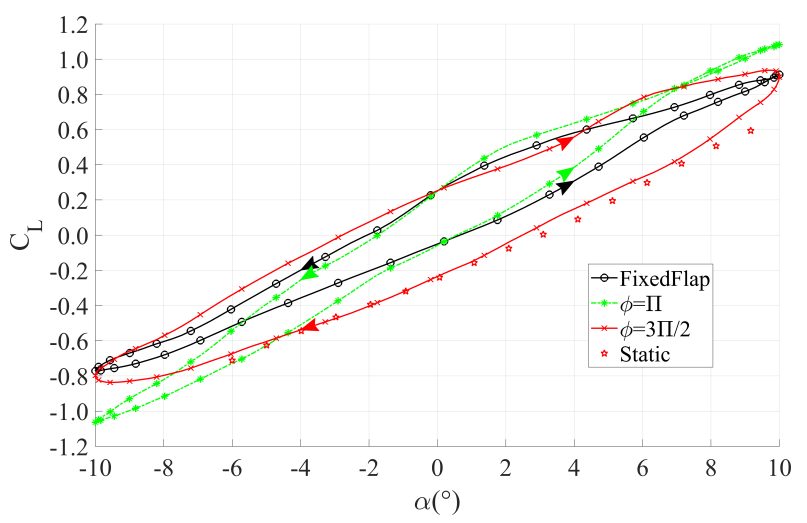

(c) Coefficient of lift $(\phi=1 \pi, \phi=3 \pi / 2)$

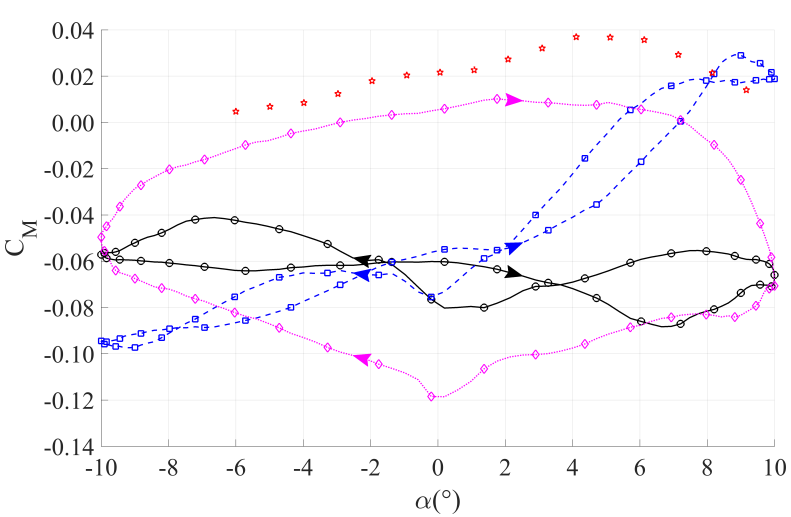

(b) Coefficient of moment $(\phi=0 \pi, \phi=\pi / 2)$

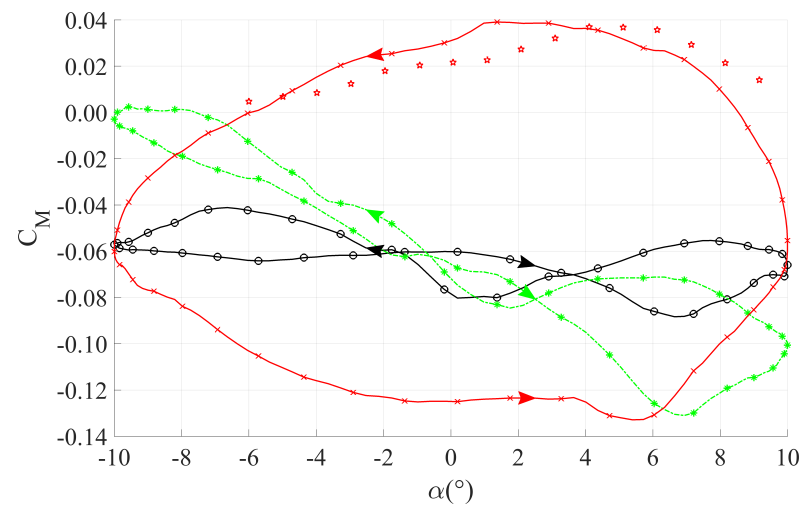

(d) Coefficient of moment $(\phi=1 \pi, \phi=3 \pi / 2)$

Fig. 8 Coefficient of lift $\left(\mathrm{C}_{\mathrm{L}}\right)$, and moment $\left(\mathrm{C}_{\mathrm{M}}\right)$ versus $\alpha$ for different flap phases $(\phi)$ for case 1a where $k=0.06$ and $\alpha_{m e a n}=0^{\circ}$. Only every fourth data point is marked on the plot for clarity.

then $\alpha_{F}=20^{\circ}$ and the camber is decreased thus reducing $\mathrm{C}_{\mathrm{L}}$. For the same curve when $\alpha=-10^{\circ}$ then $\alpha_{F}=-20^{\circ}$ and the camber is increased thus increasing $\mathrm{C}_{\mathrm{L}}$. The opposite is true when $\phi=\pi, \mathrm{C}_{\mathrm{L}}$ increases at $\alpha=10^{\circ}$ and decreases at $\alpha=-10^{\circ}$ compared to the FixedFlap curve. When $\phi=\pi / 2$ or $3 \pi / 2, \mathrm{C}_{\mathrm{L}}$ is equal to the FixedFlap at $\alpha=10^{\circ}$ and $-10^{\circ}$ due to the fact that $\alpha_{F}=0^{\circ}$ at these points. For $\phi=\pi / 2$ and $\alpha=0^{\circ}$ during the upstroke motion $\alpha_{F}=20^{\circ}$ decreasing $\mathrm{C}_{\mathrm{L}}$ but during downstroke $\alpha_{F}=-20^{\circ}$ increasing $\mathrm{C}_{\mathrm{L}}$. This indicates that when $\phi=\pi / 2$ or $3 \pi / 2$, the largest change in $\mathrm{C}_{\mathrm{L}}$ is found when $\alpha$ is near the mean angle of attack. The four $\phi$ cases presented conclude that the TEF is capable of changing $\mathrm{C}_{\mathrm{L}}$ at any point in the cycle and the variable $\phi$ determines that location of change. The airfoil and flap kinematics are illustrated in Figure 4.

The hysteresis cycle of $\mathrm{C}_{\mathrm{L}}$ is counter-clockwise (ccw) for the majority of $\phi$ values indicating that during downstroke, the lift force is higher than the upstroke for the same $\alpha$. The ccw cycle is due to the nature of dynamic motion and a characteristic of the airfoil. For example, Raiola et al. [21] tested a symmetric airfoil, the NACA 0015, under similar conditions and the results show that the hysteresis cycle was clockwise. The NACA 0015 is a symmetric airfoil while the S833 airfoil has camber and is thicker. On the other hand, for dynamic cases of cambered airfoils, the hysteresis cycle 
is counter-clockwise similar to the results presented in [15, 48, 49]. The counter-clockwise hysteresis cycle could be attributed to a vortex being generated at the LE at higher $\alpha$ that increases lift during downstroke. The most interesting case is when $\phi=3 \pi / 2$, where the direction of the hysteresis cycle is now clockwise (cw). This occurs because when $\alpha=0^{\circ}$ and during the upstroke motion, $\alpha_{F}=-20^{\circ}$ increasing $\mathrm{C}_{\mathrm{L}}$ but during downstroke $\alpha_{F}=20^{\circ}$ decreasing $\mathrm{C}_{\mathrm{L}}$. So during downstroke, $\mathrm{C}_{\mathrm{L}}$ is lower than upstroke just for $\phi=3 \pi / 2$.

The TEF also has a significant influence on $\mathrm{C}_{\mathrm{M}}$ as seen in Figures $8 \mathrm{~b}$ and $8 \mathrm{~d}$ for the different $\phi$ cases. For the FixedFlap case, the variation in $\mathrm{C}_{\mathrm{M}}$ is the lowest when compared to other flap phases. Any motion of the TEF at any $\phi$ will lead to either an increase in $C_{M}$ hysteresis or an increase in min/max $C_{M}$ peaks. In most cases, $C_{M}$ is much lower than the measured static case indicating that the airfoil is in constant nose-down pitching moment. For the FixedFlap, $\phi=0 \pi$ and $\phi=1 \pi$ cases, there are some $\mathrm{cw}$ and $\mathrm{ccw}$ loops occurring in the $\mathrm{C}_{\mathrm{M}}$ hysteresis curve. The $\mathrm{cw}$ loops in the cases mentioned occupy a small portion of the cycle but nonetheless they lead to negative damping. These small cw loops do not resemble flutter because there is no dynamic stall in this case. Dynamic stall only occurs when the airfoil pitching exceeds the static stall $\alpha$ by few degrees [4, 5], so in this case $\left(\alpha_{\text {mean }}=0^{\circ}\right)$ there should be no dynamic stall. The entirety of the $\phi=\pi / 2$ loop is $\mathrm{cw}$ producing negative damping that introduces the possibility of aeroelastic problems but may never materialize due to the lack of dynamic stall. On the other hand, for $\phi=3 \pi / 2$ the entire loop is $\mathrm{ccw}$ eliminating any possibility of flutter or aeroelastic problems due to the lack of negative damping.

Figure 9 presents $\mathrm{C}_{\mathrm{L}}$ and $\mathrm{C}_{\mathrm{M}}$ for case $1 \mathrm{~b}$ where $k$ increased from 0.06 to 0.1 and $\alpha_{\text {mean }}=0^{\circ}$. Since there is no occurrence of dynamic stall when $\alpha_{\text {mean }}=0^{\circ}$ then increasing $k$ does not create a large change in the $\mathrm{C}_{\mathrm{L}}$ and $\mathrm{C}_{\mathrm{M}}$ cycles. The major difference between case $1 \mathrm{a}$ and $1 \mathrm{~b}$ (increasing $k$ from 0.06 to 0.1 ) is that the difference between the upstroke and downstroke is wider and $\mathrm{C}_{\mathrm{L}}$ and $\mathrm{C}_{\mathrm{M}}$ increases by about $6 \%$ in extreme values. These differences are due only to an increase in $k$ as all other variables are the same as the previous case. This concludes that $k$ has great influence over the aerodynamic behavior of the flow around the airfoil and that is in alignment with what is presented in Leishman [6] and Masdari et al.[15].

A contour plot of $\Delta \mathrm{C}_{\mathrm{p}}$ is presented in Figure 10 where the chord length is plotted on the $\mathrm{x}$-axis and the period of the averaged cycle is plotted on the y-axis for case $1 \mathrm{~b}\left(k=0.1\right.$ and $\left.\alpha_{\text {mean }}=0^{\circ}\right)$. The plots are a matrix of 27 differential pressure transducers representing the airfoil chord by 160 bins representing 1 complete period. Each point in the matrix is the average of 4000 data points. $t / \tau$ represents a dimensionless time unit to represent a cycle ( $\tau$ is the period of the cycle). Arrows along the $y$-axis indicate upstroke or downstroke in the cycle. $\mathrm{t} / \tau$ could also be associated with $\alpha$ as shown on the right y-axis and the relationship is plotted in Figure $10 \mathrm{~d}$ for $\phi=\pi$. The contour plot is not symmetric about $\mathrm{t} / \tau=0.5$ and the $-\Delta \mathrm{C}_{\mathrm{p}}$ peak is shifted slightly up. This shift is likely due to the nature of having unsteady flow while pitching. The unsteady flow can create a phase lag between the fluid reaction and the pitching motion [4]. This $-\Delta \mathrm{C}_{\mathrm{p}}$ peak shift leads to higher lift values during the downstroke when compared to the upstroke. This results in the ccw direction of the $C_{L}$ hysteresis cycle mentioned in Figures 8 and 9 . Comparing $\phi=0 \pi$ (Figure $10 \mathrm{~b}$ ) to the 


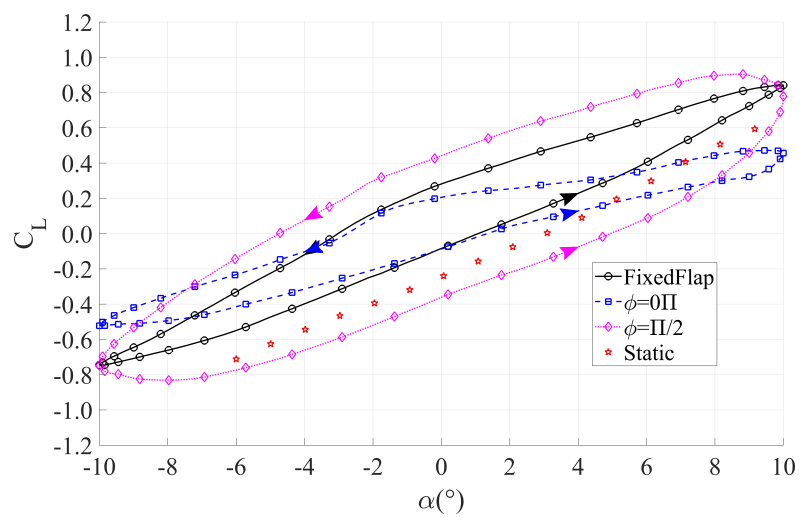

(a) Coefficient of lift $(\phi=0 \pi, \phi=\pi / 2)$

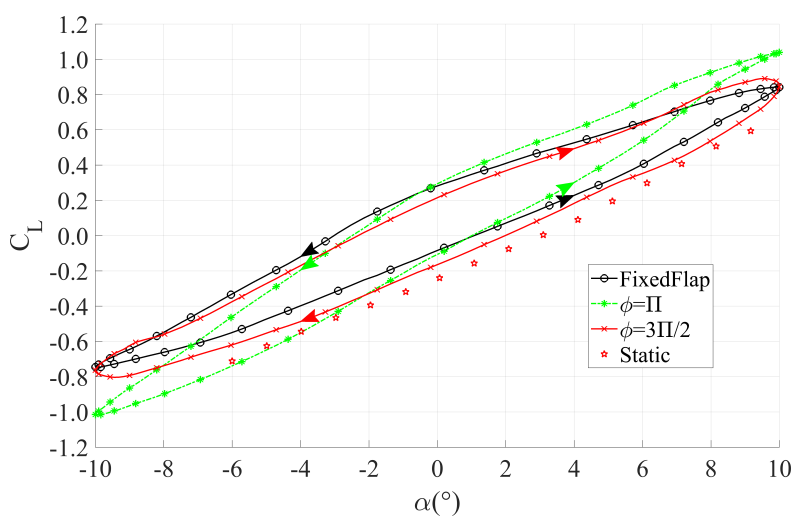

(c) Coefficient of lift $(\phi=1 \pi, \phi=3 \pi / 2)$

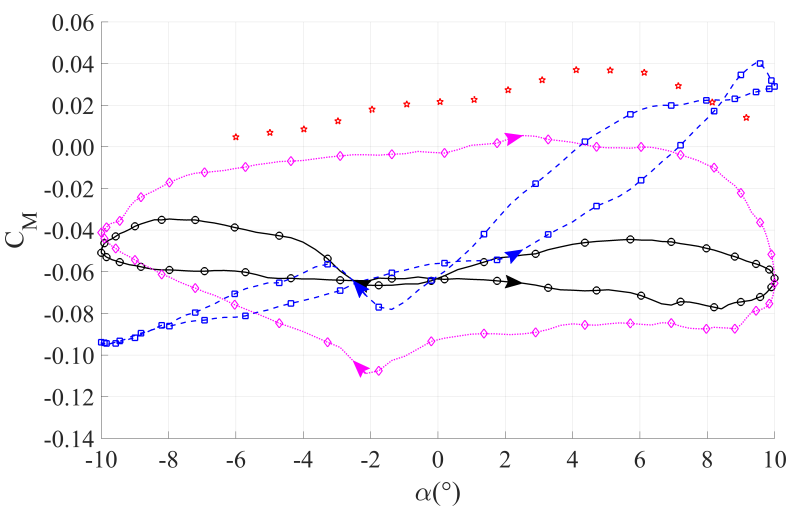

(b) Coefficient of moment $(\phi=0 \pi, \phi=\pi / 2)$

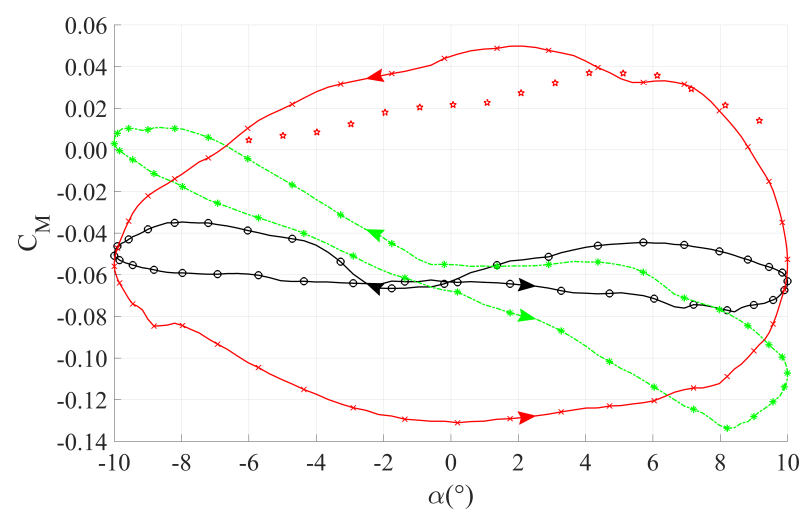

(d) Coefficient of moment $(\phi=1 \pi, \phi=3 \pi / 2)$

Fig. 9 Coefficient of lift $\left(\mathrm{C}_{\mathrm{L}}\right)$, and moment $\left(\mathrm{C}_{\mathrm{M}}\right)$ versus $\alpha$ for different flap phases $(\phi)$ for case $1 \mathrm{~b}$ where $k=0.1$ and $\alpha_{m e a n}=0^{\circ}$. Only every fourth data point is marked on the plot for clarity.

FixedFlap case (Figure $10 \mathrm{p}$ ), the $-\Delta \mathrm{C}_{\mathrm{p}}$ values are lower and the constant $-\Delta \mathrm{C}_{\mathrm{p}}$ iso-lines are shifted towards the $\mathrm{LE}$. When $\mathrm{t} / \tau=0.5$ and $\phi=0 \pi$ then $\alpha_{F}$ would be $20^{\circ}$ decreasing airfoil camber and decreasing the $-\Delta \mathrm{C}_{\mathrm{p}}$ to negative values close to the TE. This indicates that there is negative $-\Delta \mathrm{C}_{\mathrm{p}}$ (suction side pressure higher than pressure side) around the TEF area thus leading to a nose-up pitching moment as indicated in Figures 8 and 9 Now comparing $\phi=1 \pi$ (Figure 10k) to the FixedFlap case (Figure $10 \mathrm{p}$ ) the $-\Delta \mathrm{C}_{\mathrm{p}}$ values are higher and the constant $-\Delta \mathrm{C}_{\mathrm{p}}$ iso-lines are shifted away from the LE. Focusing on $\mathrm{t} / \tau=0.5$ again, when $\phi=1 \pi$, then $\alpha_{F}=-20^{\circ}$. This increases $-\Delta \mathrm{C}_{\mathrm{p}}$ values close to the TE and leads to a nose-down pitching moment. 

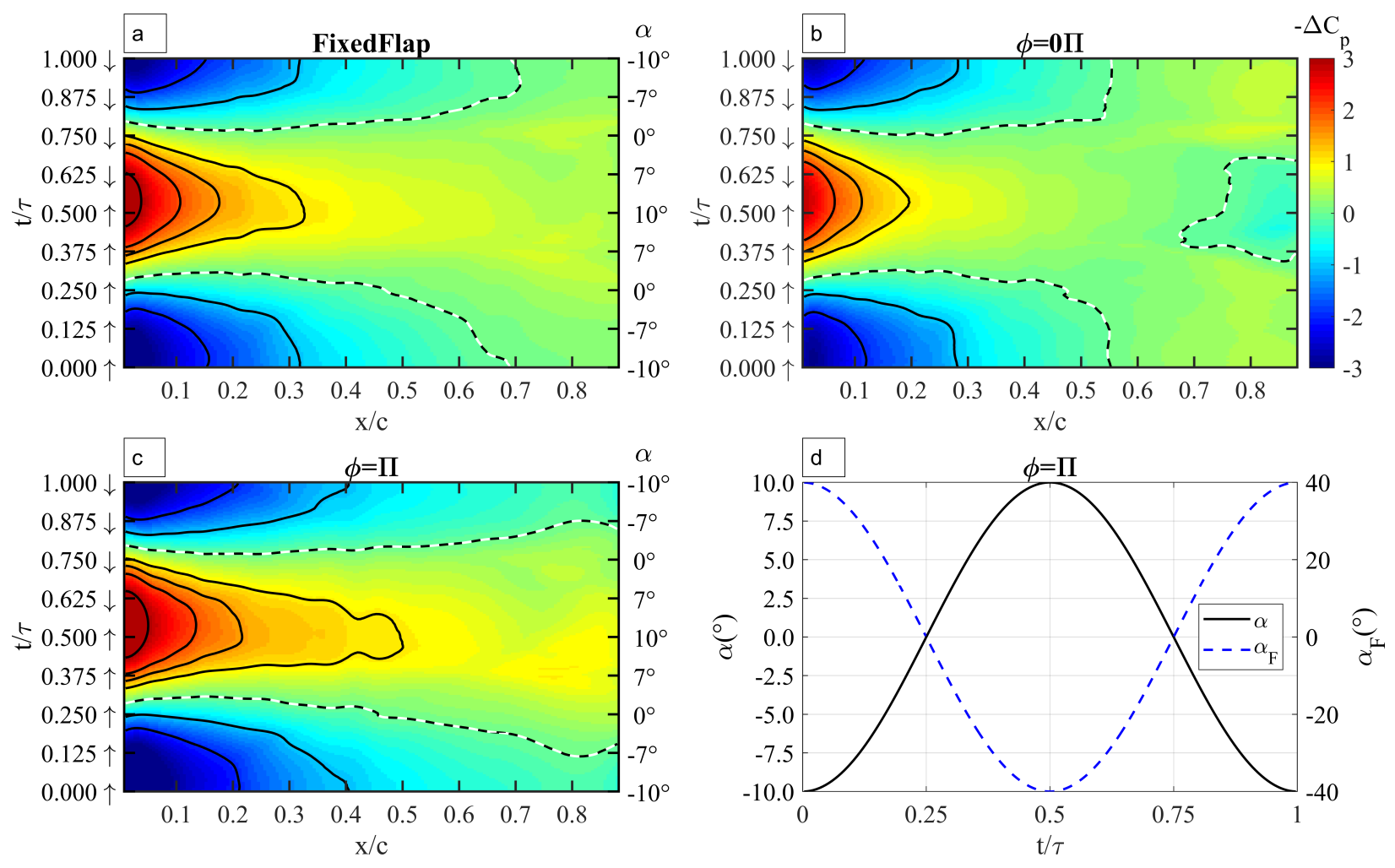

Fig. 10 Contours of $-\Delta \mathrm{C}_{\mathrm{p}}$ versus $\mathrm{x} / \mathrm{c}$ versus $\alpha$ for case $1 \mathrm{~b}\left(k=0.1\right.$ and $\left.\alpha_{\text {mean }}=0^{\circ}\right)$. a), b), and c) represent FixedFlap, $\phi=0 \pi$, and $\phi=1 \pi$ respectively. The angle of attack $(\alpha)$ is shown on the secondary axis. d) $\alpha$ versus pitch period $(\mathrm{t} / \tau)$, and flap angle $\left(\alpha_{F}\right)$ for phase $\phi=1 \pi$. The iso-lines on the plot represent constant $-\Delta \mathrm{C}_{\mathrm{p}}$ values $=[-2,-1,0,1,1.5,2,3]$.

The $\Delta \mathrm{C}_{\mathrm{p}}$ standard deviation (STD) contour plots for the same case, $1 \mathrm{~b}$, are shown in Figure 11 The contour plots are plotted in $\log _{10}$ scale to better show the details at lower STD values. The transition point from laminar to turbulent flow is determined by locating the maximum value of the STD at each specific point of time along the chord as has been done by Raiola et al. [21]. A dashed line is added to highlight the location of the transition point. The pattern highlighted has a frequency twice that of the flapping motion because the transition occurs on both sides of the airfoil. Each side of the airfoil acts as both a pressure and suction side. Pressure fluctuations are higher around $\mathrm{t} / \tau \approx 0.25$ and 0.75 when $\alpha=0^{\circ}$ because the suction and pressure side switch sides. In Figure $11 \mathrm{c}$ the $\operatorname{STD}\left(\Delta \mathrm{C}_{\mathrm{p}}\right)$ is high around $\mathrm{t} / \tau \approx 0.5$ and $\mathrm{x} / \mathrm{c}>0.7$ because $\alpha_{F}=-20^{\circ}$ and the camber of the airfoil is at its highest. When $\alpha_{F}=-20^{\circ}$ it creates a high angle that forces the airflow close to the TE to separate and this increases STD. 

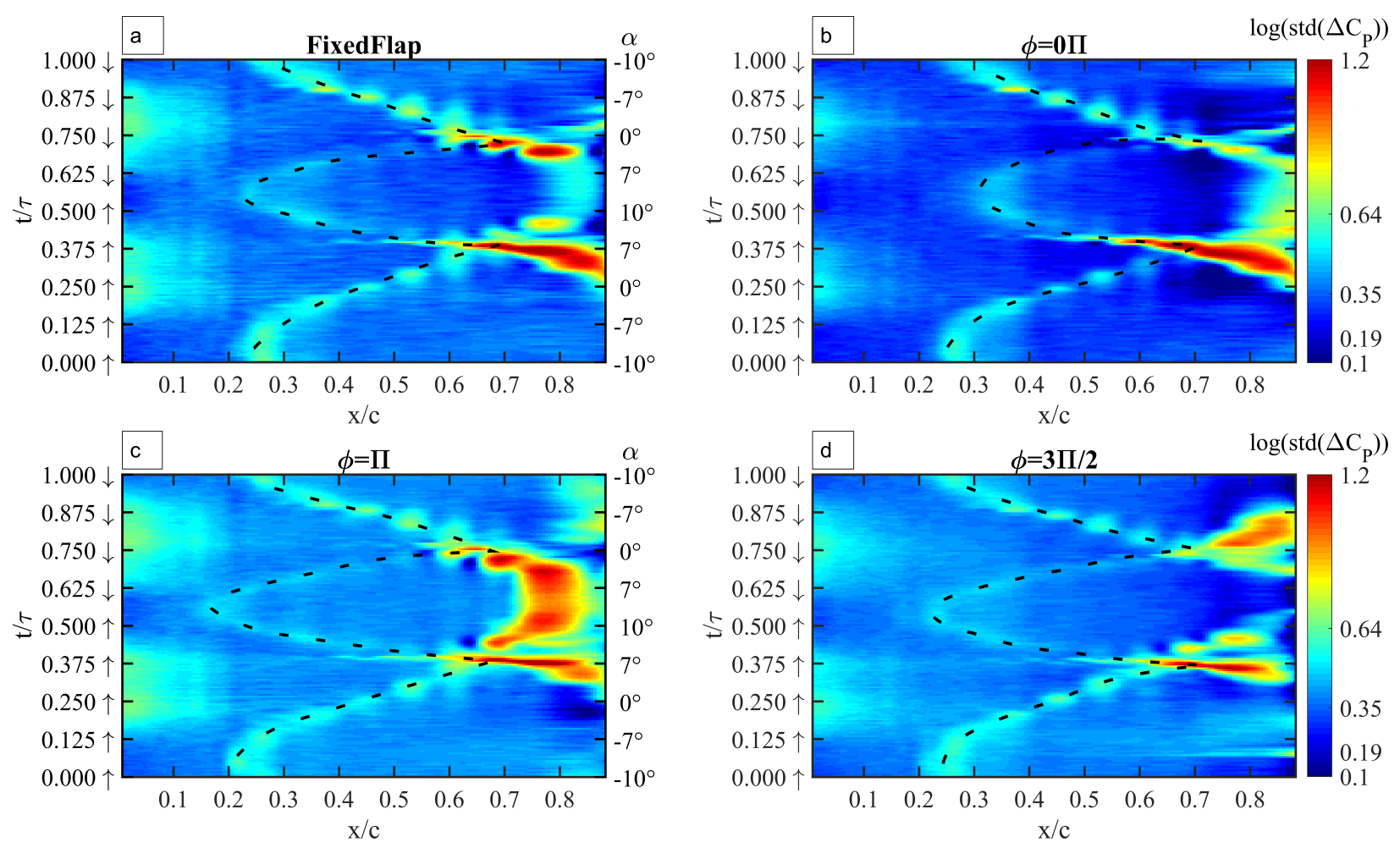

Fig. 11 Contours of $\operatorname{STD}\left(\Delta \mathrm{C}_{\mathrm{p}}\right)$ versus $\mathrm{x} / \mathrm{c}$ versus $\mathrm{t} / \tau$ for case $1 \mathrm{~b}\left(k=0.1\right.$ and $\left.\alpha_{\text {mean }}=0^{\circ}\right)$. a), b), c), and d) represent FixedFlap, $\phi=0 \pi, \phi=1 \pi$, and $\phi=3 \pi / 2$ respectively. The angle of attack $(\alpha)$ is shown on the secondary axis. The dashed line shows the location of the transition point.

2. $\alpha_{\text {mean }}=10^{\circ}$

The same figures in section III.B.1 are also presented for cases $2 \mathrm{a}$ and $2 \mathrm{~b}$ in this section where the only experimental parameter difference is changing $\alpha_{\text {mean }}$ from $0^{\circ}$ to $10^{\circ}$. The change of $\alpha_{\text {mean }}$ alters the cycle loop significantly especially at high $\alpha$ values. The $\mathrm{C}_{\mathrm{L}}$ values are approximately double their static counterparts when $18^{\circ}<\alpha<20^{\circ}$ due to dynamic stall occurrence. The static lift measurements are marked for reference in red stars $(\star)$ in the same plot. Looking at $\mathrm{C}_{\mathrm{L}}$ in Figures $12 \mathrm{a}$ and $12 \mathrm{c}$, a similar trend can be seen across the different flap phases but with a differing magnitude and location. Starting at $\alpha=0^{\circ}$ and for all cases, as the airfoil pitches upward, $\mathrm{C}_{\mathrm{L}}$ keeps increasing past the static stall angle. When $\alpha$ is between $17^{\circ}$ and $19^{\circ}$ there is a sudden sharp increase in $\mathrm{C}_{\mathrm{L}}$. This is likely due to a LEV generation and shedding from the LE as explained in the introduction and noted by Corke and Thomas [5]. The LEV creates extra suction on the suction side of the airfoil enhancing $C_{L}$. While comparing the $\mathrm{C}_{\mathrm{L}}$ cycles for the different $\phi$ cases, it was concluded that the TEF is not capable of controlling the formation of the LEV but rather it delays or promotes the formation of the LEV as was concluded by Lee and Gerontakos [10]. For example, compared to the FixedFlap when $\phi=\pi$ the sharp increase in $\mathrm{C}_{\mathrm{L}}$ (or LEV) occurs $1^{\circ}$ earlier and when $\phi=0 \pi$ the sharp increase in $\mathrm{C}_{\mathrm{L}}$ occurs $0.5^{\circ}$ later. As soon as the airfoil pitching reaches the top of the upstroke cycle and just before reversing direction $\mathrm{C}_{\mathrm{L}}$ drops dramatically due to shedding of the LEV. After the primary LEV is shed, a second $\mathrm{C}_{\mathrm{L}}$ peak occurs created by 
a secondary LEV which is weaker in magnitude. The secondary LEV occurs during the upstroke motion in some cases, and in other cases occurs during the downstroke motion. The formation of multiple LEV is reported in the literature review by Leishman [6] and Gharali and Johnson [7]. During stall and after the secondary LEV, $\mathrm{C}_{\mathrm{L}}$ keeps decreasing as $\alpha$ decreases. The airfoil is stalled even though $\alpha$ is below the stall point which is a characteristic of dynamic stall that delays flow reattachment. At around $8^{\circ}$ and during the downstroke cycle, $\mathrm{C}_{\mathrm{L}}$ increases to a higher value than the static $\mathrm{C}_{\mathrm{L}}$ plot indicating that the flow is starting to reattach starting from the LE. Flow reattachment after stall is more clearly seen in the $\operatorname{STD}\left(\Delta \mathrm{C}_{\mathrm{p}}\right)$ contour plots in Figure 15 and discussed later in this section.

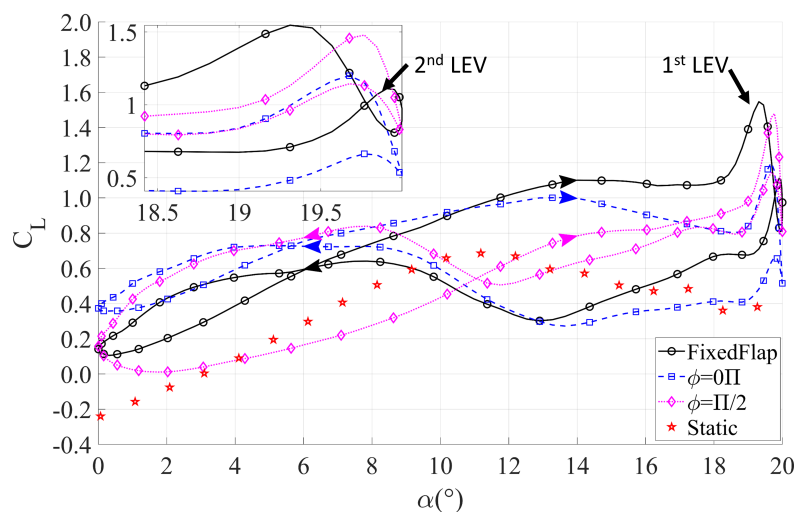

(a) Coefficient of lift $(\phi=0 \pi, \phi=\pi / 2)$

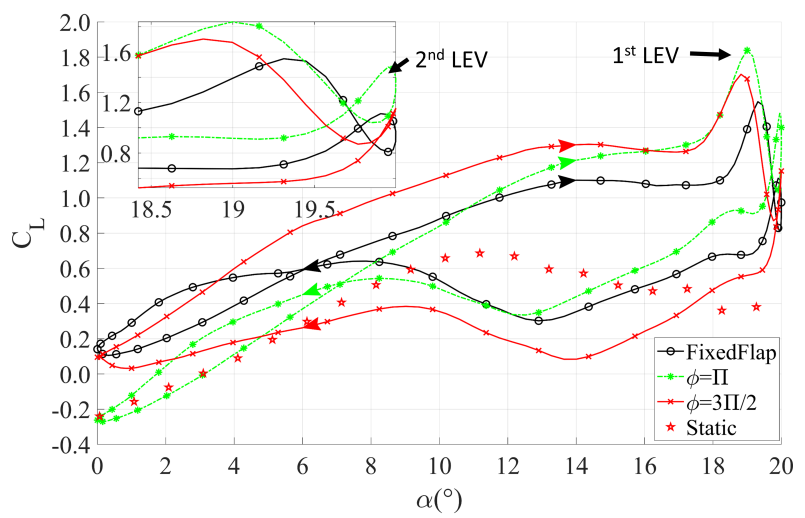

(c) Coefficient of lift $(\phi=1 \pi, \phi=3 \pi / 2)$

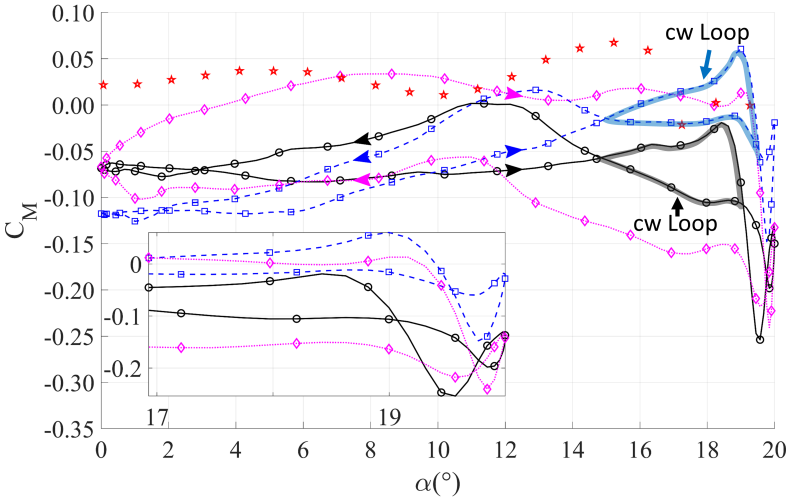

(b) Coefficient of moment $(\phi=0 \pi, \phi=\pi / 2)$

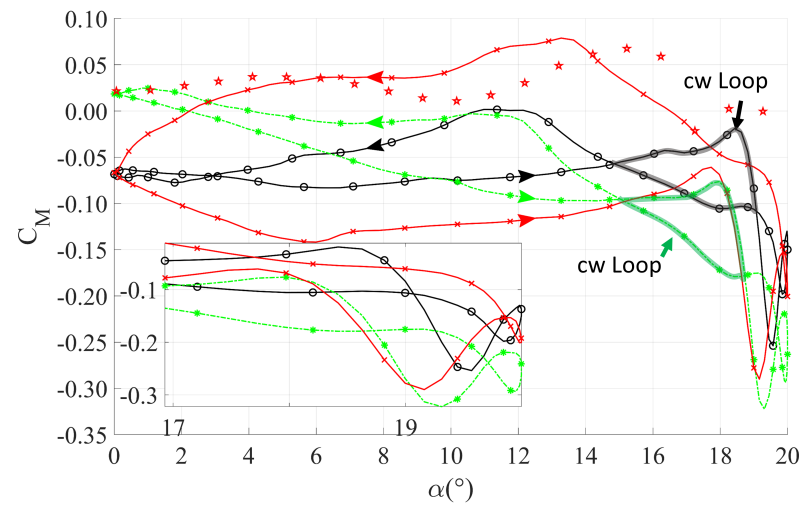

(d) Coefficient of moment ( $\phi=1 \pi, \phi=3 \pi / 2$ )

Fig. 12 Coefficient of lift $\left(\mathrm{C}_{\mathrm{L}}\right)$, and moment $\left(\mathrm{C}_{\mathrm{M}}\right)$ versus $\alpha$ for different flap phases $(\phi)$ for case $2 \mathrm{a}$ where $k=0.06$ and $\alpha_{m e a n}=10^{\circ}$. Only every fourth data point is marked on the plot for clarity. A plot inset is used to magnify the data around $\alpha=19^{\circ}$. The $1^{s t}$ and $2^{\text {nd }}$ LEV and the cw loops are marked in each plot.

For all cases except when $\phi=3 \pi / 2$ in Figures $12 \mathrm{a}$ and $12 \mathrm{c}$, the $\mathrm{C}_{\mathrm{L}}$ loop crosses over itself half way through the downstroke. This is also seen in other experimental work [15, 46]. When the loop crosses over, it indicates that during upstroke the $\mathrm{C}_{\mathrm{L}}$ is lower than downstroke. This is in agreement with case 1a $\left(\alpha_{\text {mean }}=0^{\circ}\right)$ where the loop direction is also ccw. For $\alpha<6^{\circ}$ and case $2 \mathrm{a}$, the $\mathrm{C}_{\mathrm{L}}$ variation in magnitude and shape is similar to case 1a. Looking at the overall $\mathrm{C}_{\mathrm{L}}$ variation it could be noted that when $\phi=\pi$ the lift force fluctuation is the highest while when $\phi=0 \pi$ the lift force fluctuation is the lowest. In both cases $(\phi=0 \pi$ and $\pi$ ), the variation around $\alpha=0$ is negligible when compared to the 
FixedFlap case. For $\phi=\pi / 2$ and $\phi=3 \pi / 2$, the difference between the upstroke and downstroke is narrower and wider respectively. Based on these four $\phi$ phases, it could be concluded that the TEF is capable of reducing or increasing the $\mathrm{C}_{\mathrm{L}}$ at any specific point along the dynamic stall cycle based on the value of $\phi$.

Figures $12 \mathrm{~b}$ and $12 \mathrm{~d}$ show the variation in $\mathrm{C}_{\mathrm{M}}$ for different flap phases for case $2 \mathrm{a}$. For $\alpha<12^{\circ}$ the variations in $\mathrm{C}_{\mathrm{M}}$ for all $\phi$ cases are relatively flat and on the same scale as in case $1 \mathrm{a}$ with $\alpha_{\text {mean }}=0^{\circ}$. The $\mathrm{C}_{\mathrm{M}}$ static values are also shown in the plot for comparison. During the upstroke motion (for all $\phi$ cases) and just when the pitching motion is about to reverse, the LEV formation and shedding causes a rapid and severe increase in nose-down pitching moment (negative $\mathrm{C}_{\mathrm{M}}$ ) at about $19^{\circ}$. The peak in $\mathrm{C}_{\mathrm{M}}$ always occurs after the peak in $\mathrm{C}_{\mathrm{L}}$. The vortex starts at the $\mathrm{LE}$ and passes along the airfoil exiting at the TE. While the vortex sweeps over the airfoil, it produces a rapid aft movement of the center of pressure from the LE towards the TE resulting in a large nose-down pitching moment on the airfoil [16].

Another important factor to look for in the $\mathrm{C}_{\mathrm{M}}$ plots is the formation of negative damping as discussed in the introduction. Negative damping occurs when $\mathrm{C}_{\mathrm{M}}-\alpha$ creates a clockwise (cw) cycle and that promotes flutter and instabilities and would not be a good choice for operation. The $\mathrm{cw}$ loop (highlighted and labeled in Figures $12 \mathrm{~b}$ and $12 \mathrm{~d}$ ) could be seen in the FixedFlap case, $\phi=0 \pi$, and $\phi=\pi$. In these particular cases the cw loops are likely to produce airfoil flutter due to the existence of dynamic stall and a LEV [5, 17]. For $\phi=\pi / 2$ the entire loop is negatively damped while $\phi=3 \pi / 2$ produces no negative damping. The TEF was capable of reducing and increasing the minimum $\mathrm{C}_{\mathrm{M}}$ peak and was also able to remove negative damping effects and reduce flutter for specific flap phases.

$\mathrm{C}_{\mathrm{L}}$ and $\mathrm{C}_{\mathrm{M}}$ are plotted in Figure 13 for case $2 \mathrm{~b}$ with a reduced frequency of 0.1 . When comparing case $2 \mathrm{~b}$ to $2 \mathrm{a}$ (Figure 12) where $k$ increased from 0.06 to 0.1 , a few significant differences arise. These differences include a rise in the $\mathrm{C}_{\mathrm{L}}$ peak indicating a larger and stronger LEV as seen in Figure $13 \mathrm{a}$ and $13 \mathrm{c}$. The LEV onset is also delayed by $1^{\circ}$ pushing the peak location to the right from $19^{\circ}$ (case 2a) to $20^{\circ}$ (case 2b). The secondary LEV onset is also delayed by $2^{\circ}$ and occurs at about $18^{\circ}$ during the downstroke. Increasing $k$ is then found to delay the onset of flow separation and dynamic stall to a higher $\alpha$. Reattachment is also delayed by $4^{\circ}$. The difference between the upstroke and downstroke is larger slightly widening the hysteresis loop. Comparing $\mathrm{C}_{\mathrm{M}}$ for case $2 \mathrm{~b}$ (Figure 13) against case 2a (Figure 12) a few notable changes are worth mentioning. The negative damping loops are longer and larger in magnitude and negative damping now occurs for $\phi=3 \pi / 2 . \phi=\pi / 2$ is the only case where negative damping occurs during the entire cycle. It is found that when $k$ increased from 0.06 to 0.1 , the negative damping loop increased.

Now that the plots for cases $2 \mathrm{a}$ and $2 \mathrm{~b}$ are presented for different $\phi$ cases, a conclusion could be made about the influence of $\phi$. If the aim is to reduce the $\mathrm{C}_{\mathrm{L}}$ fluctuations around the mean angle then it would be best to set $\phi=\pi / 2$. On the other hand, if the aim is to reduce $\mathrm{C}_{\mathrm{L}}$ fluctuations at the min and max then it would be best to set $\phi=0 \pi$. Now focusing on the $\mathrm{C}_{\mathrm{M}}$ plots, it was found that $\phi=0 \pi$ decreases the peaks and reduces the negative damping loop in size. For $\phi=\pi / 2$ it was found that the entire $\mathrm{C}_{\mathrm{M}}$ loop causes negative damping possibly promoting stall flutter. It is recommended then to use $\phi=0 \pi$ because it reduces the $\min / \max$ of the $\mathrm{C}_{\mathrm{L}}$ and $\mathrm{C}_{\mathrm{M}}$ cycle and reduces negative 
damping.

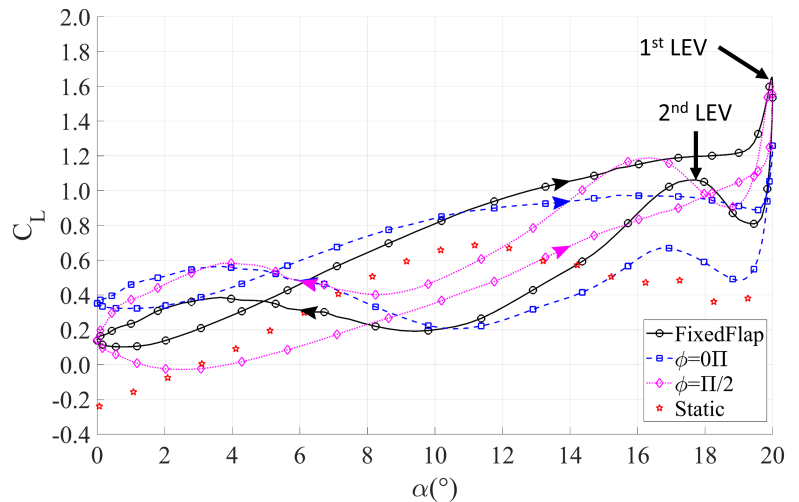

(a) Coefficient of lift $(\phi=0 \pi, \phi=\pi / 2)$

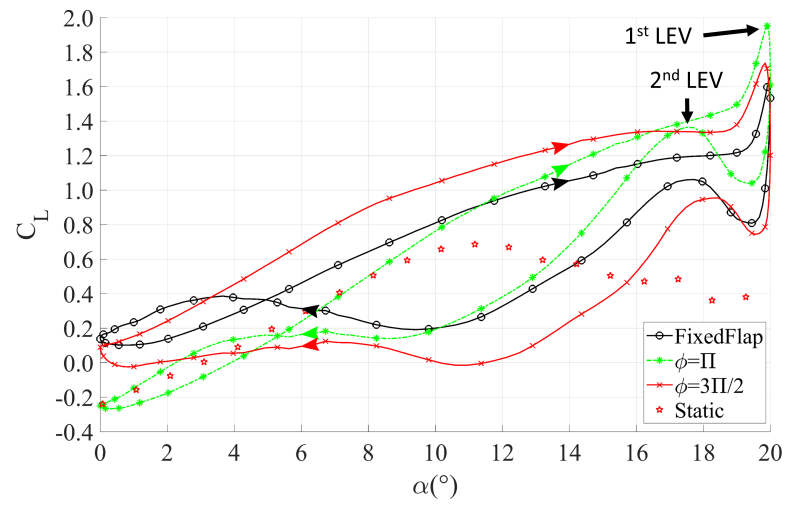

(c) Coefficient of lift $(\phi=1 \pi, \phi=3 \pi / 2)$

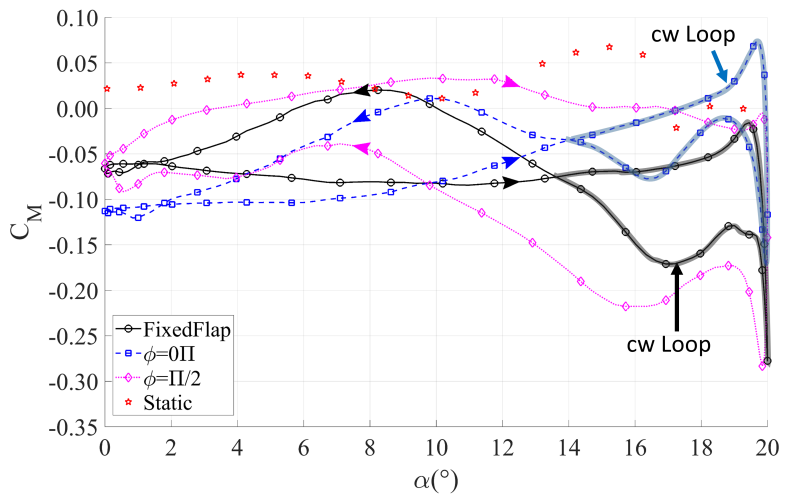

(b) Coefficient of moment $(\phi=0 \pi, \phi=\pi / 2)$

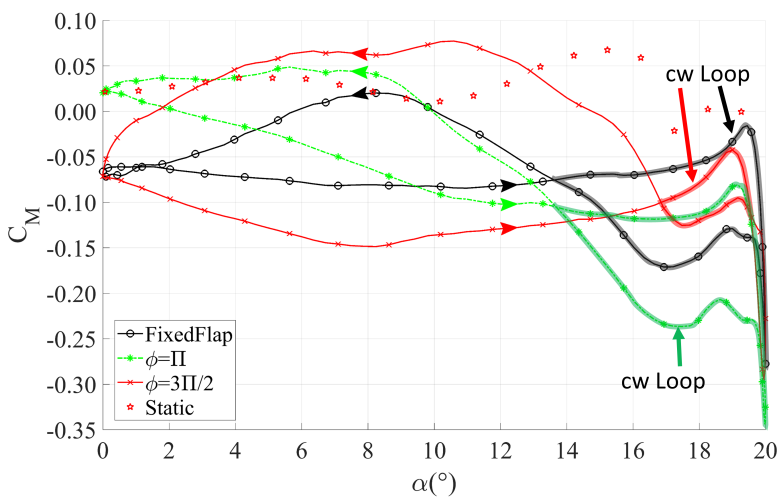

(d) Coefficient of moment $(\phi=1 \pi, \phi=3 \pi / 2)$

Fig. 13 Coefficient of lift $\left(\mathrm{C}_{\mathrm{L}}\right)$, and moment $\left(\mathrm{C}_{\mathrm{M}}\right)$ versus $\alpha$ for different flap phases $(\phi)$ for case $2 \mathrm{~b}$ where $k=0.1$ and $\alpha_{\text {mean }}=10^{\circ}$. Only every fourth data point is marked on the plot for clarity. The $1^{\text {st }}$ and $2^{\text {nd }}$ LEV and the cw loops are marked in each plot.

To understand the importance of the formation and shedding of the vortices, the temporal change in $\Delta \mathrm{C}_{\mathrm{p}}$ is plotted in Figure 14 for case $2 \mathrm{~b}$ just as case $1 \mathrm{~b}$ was plotted in Figure $10, \Delta \mathrm{C}_{\mathrm{p}}$ for case $2 \mathrm{a}$ is not shown here because the trends are very similar. The FixedFlap case, $\phi=0 \pi$ and $\phi=1 \pi$ are presented along with a plot showing the correlation between the period $(\tau)$ and $\alpha$ and $\alpha_{F}$ for $\phi=1 \pi$. During the upstroke motion (as indicated with the arrows on the left axis) and for all cases, a high $-\Delta \mathrm{C}_{\mathrm{p}}$ peak is present at the $\mathrm{LE}$ after $\mathrm{t} / \tau=0.25$ that gets stronger with increasing $\alpha$. When the pitch motion is about to reverse, the LEV is shed increasing $-\Delta \mathrm{C}_{\mathrm{p}}$ along the entire chord. This is seen in the plots at $\mathrm{t} / \tau=0.45$ where the LEV is clearly seen moving from the leading to the trailing edge. Even though the $-\Delta \mathrm{C}_{\mathrm{p}}$ peak at the $\mathrm{LE}$ is decreased, $-\Delta \mathrm{C}_{\mathrm{p}}$ on the entire chord increases thus increasing the total lift as indicated in the $\mathrm{C}_{\mathrm{L}}$ versus $\alpha$ plots in Figures 12 and 13 It is seen in the contour plots that as the vortex sweeps over the airfoil, it produces a rapid aft movement of the center of pressure from the LE towards the TE resulting in a large nose-down pitching moment on the airfoil. After the LEV is shed indicated by a decrease in $-\Delta \mathrm{C}_{\mathrm{p}},-\Delta \mathrm{C}_{\mathrm{p}}$ increases slightly at $\mathrm{t} / \tau=0.625$ indicating the formation of the secondary LEV. Full reattachment takes place at about $t / \tau=0.75$ where the highest suction is located at 
the LE. The major difference between the three plots is the variation in $-\Delta \mathrm{C}_{\mathrm{p}}$ magnitude for the different flap phases. The constant $-\Delta \mathrm{C}_{\mathrm{p}}$ iso-lines clearly show how $\phi$ influences the pressure over the airfoil. The magnitude of the LEV is decreased for $\phi=0 \pi$ and increased for $\phi=\pi$ and that is in agreement with $\mathrm{C}_{\mathrm{L}}-\alpha$ (Figures 12 and 13 ). To conclude, these plots clearly show the temporal change of $-\Delta \mathrm{C}_{\mathrm{p}}$ and clearly show the importance of the LE region and the impact of the dynamic motion.
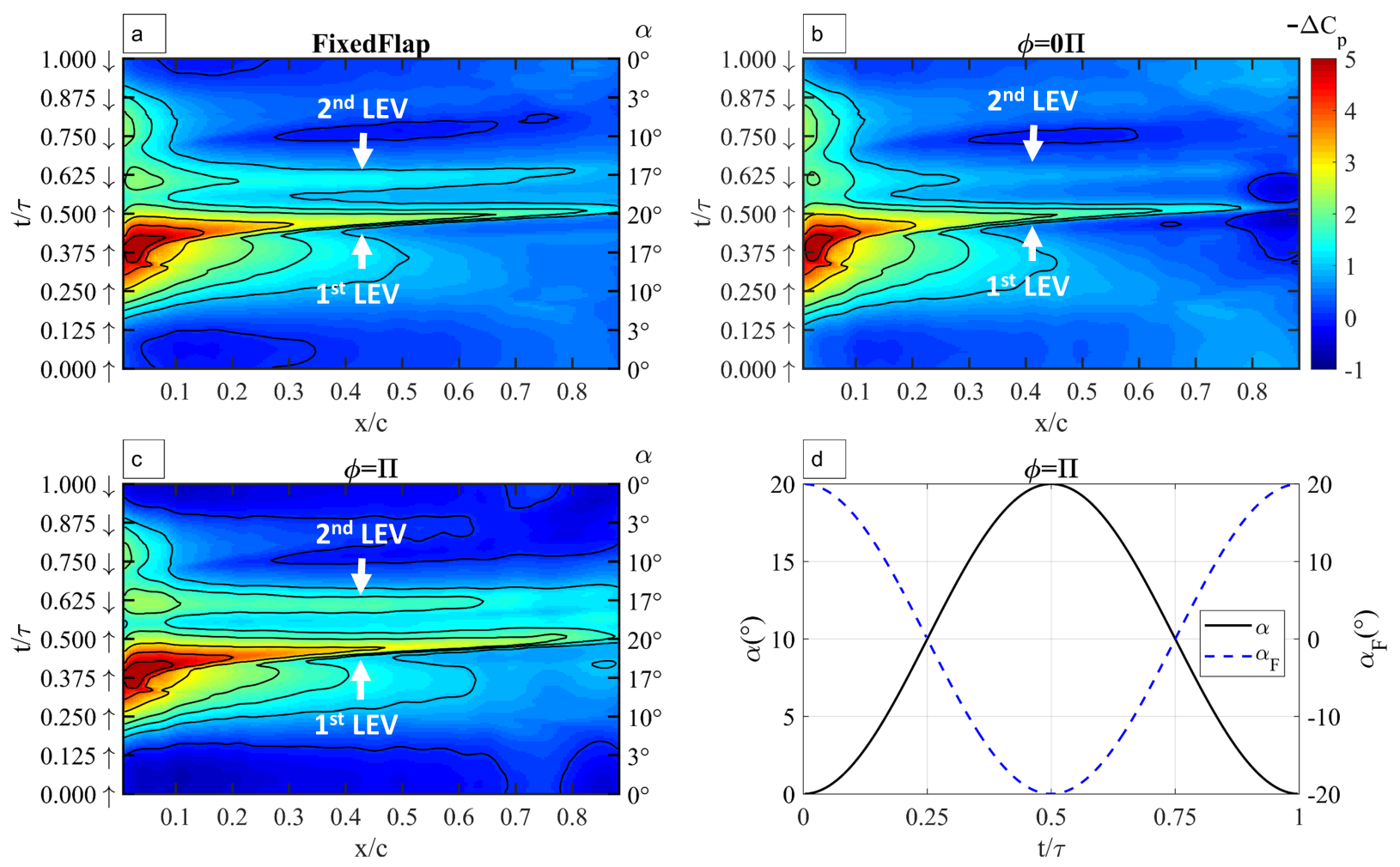

Fig. 14 Contours of $-\Delta \mathrm{C}_{\mathrm{p}}$ versus $\mathrm{x} / \mathrm{c}$ versus $\alpha$ for case $2 \mathrm{~b}\left(k=0.1\right.$ and $\left.\alpha_{\text {mean }}=10^{\circ}\right)$. a), b), and c) represent FixedFlap, $\phi=0 \pi$, and $\phi=1 \pi$ respectively. d) shows $\alpha$ versus the pitch period, $\mathrm{t} / \tau$, and flap angle, $\alpha_{F}$, for phase $\phi=1 \pi$. The angle of attack $(\alpha)$ is shown on the secondary axis. The iso-lines on the plot represent constant $-\Delta \mathrm{C}_{\mathrm{p}}$ values $=[-1,0,1,1.5,2,3,4,5]$. The $1^{\text {st }}$ and $2^{\text {nd }}$ LEV are marked in each plot.

Another way to visualize airfoil stall and vortices is by looking at the standard deviation in $\Delta \mathrm{C}_{\mathrm{p}}$ in Figure 15 for case $2 \mathrm{~b}$. The STD is plotted in $\log _{10}$ scale to show the small changes at lower values. Vortices and stall are associated with high pressure fluctuations and can be clearly seen in the figure. The high $\operatorname{STD}\left(\Delta \mathrm{C}_{\mathrm{p}}\right)$ streak at $\mathrm{t} / \tau=0.45$ is associated with the LEV being shed and the direction of movement is from the LE to the TE because the pressure center in the plot moves from $x / c=0$ to $x / c=1$ and at the same time moves up along the $y$-axis. At $t / \tau=0.5$ the magnitude decreases indicating stall but then increases again at $t / \tau=0.625$ indicating the formation of the secondary LEV. After the secondary LEV, the airfoil is completely stalled until $\mathrm{t} / \tau \approx 0.75$ where the $\operatorname{STD}\left(\Delta \mathrm{C}_{\mathrm{p}}\right)$ is greatly reduced indicating airflow re-attachment on the airfoil. The patterns in $\operatorname{STD}\left(\Delta \mathrm{C}_{\mathrm{p}}\right)$ do not change significantly for the different flap phases but rather just the magnitude. 

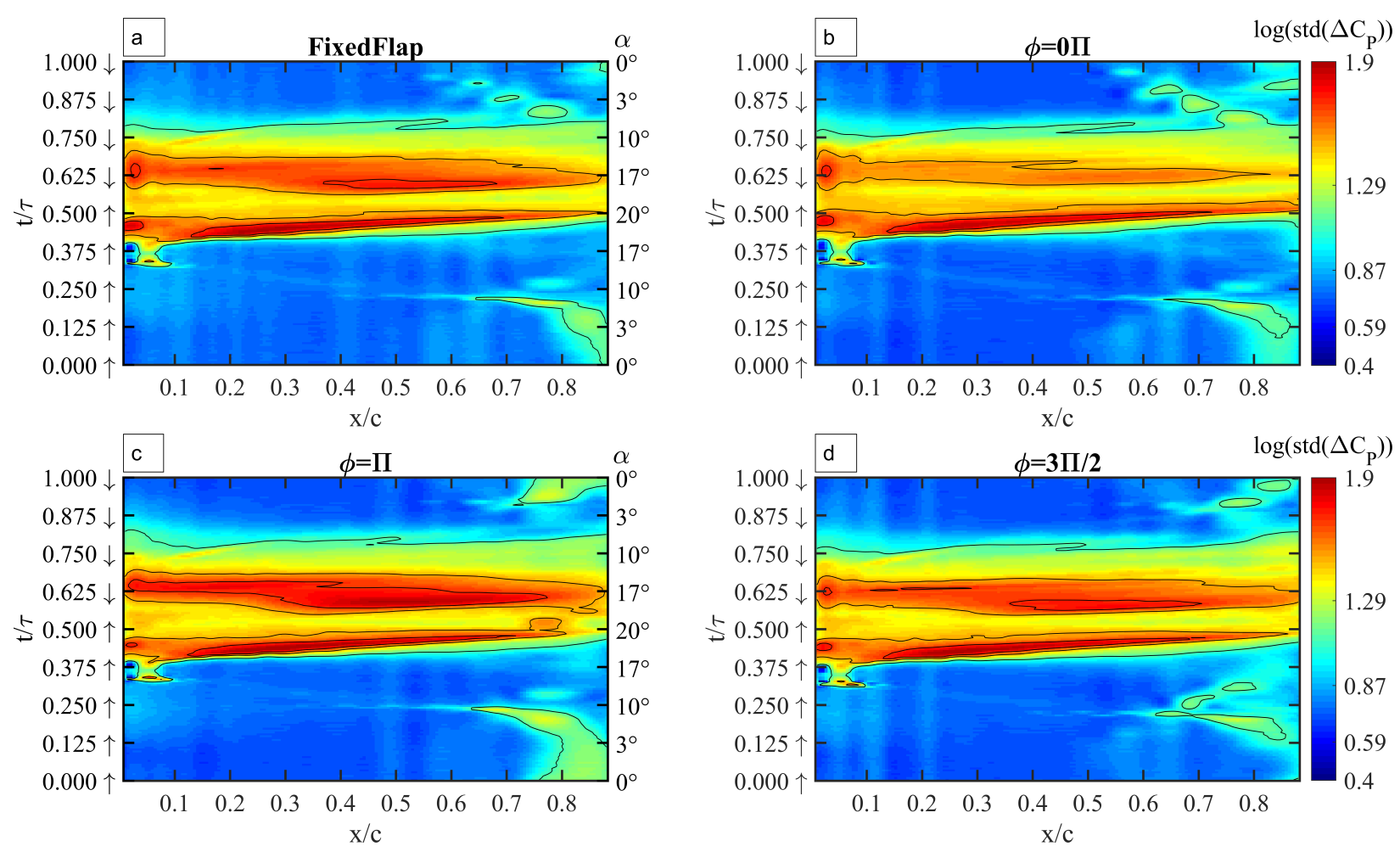

Fig. 15 Contours of $\log \left(\operatorname{STD}\left(\Delta \mathrm{C}_{\mathrm{p}}\right)\right)$ versus $\mathrm{x} / \mathrm{c}$ versus $\alpha$ for case $2 \mathrm{~b}\left(k=0.1\right.$ and $\left.\alpha_{\text {mean }}=10^{\circ}\right)$. a), b), c), and d) represent FixedFlap, $\phi=0 \pi, \phi=1 \pi$, and $\phi=3 \pi / 2$ respectively. The angle of attack $(\alpha)$ is shown on the secondary axis. The iso-lines on the plot represent constant $\log \left(\operatorname{STD}\left(\Delta \mathrm{C}_{\mathrm{p}}\right)\right)$ values.

\section{Conclusions}

The static measurements of $\mathrm{C}_{\mathrm{L}}$ and $\mathrm{C}_{\mathrm{M}}$ for different flap angles and a wide range of pitch angles are briefly presented at Re of 170,000. They show that a TEF is capable of increasing and decreasing $C_{L}$ and $C_{M}$ for all the $\alpha$ range tested. After the stall point, the experimental results show that there is a temporarily loss of lift but $\mathrm{C}_{\mathrm{L}}$ starts increasing again while $\mathrm{C}_{\mathrm{M}}$ decreases producing a large nose-down pitching moment on the airfoil. The pressure data also showed that after stall, the pressure decreases in peak magnitude and is no longer concentrated close to the LE.

The hysteresis curves for $\mathrm{C}_{\mathrm{L}}$ and $\mathrm{C}_{\mathrm{M}}$ versus $\alpha$ are presented for a pitching airfoil with reduced frequencies of 0.06 and 0.1 and for $\alpha_{\text {mean }}$ of $0^{\circ}$ and $10^{\circ}$. In all cases the amplitude was set to $10^{\circ}$. For $\alpha_{\text {mean }}$ of $0^{\circ}$, the hysteresis cycle of $\mathrm{C}_{\mathrm{L}}$ is ccw due to the characteristics of the airfoil and the phase lag created by unsteady flow behavior. The TEF was however able to manipulate $\mathrm{C}_{\mathrm{L}}$ to produce a cw cycle for a specific flap motion. Since there is no occurrence of dynamic stall when $\alpha_{\text {mean }}=0^{\circ}$ then increasing $k$ does not create a large change in the $\mathrm{C}_{\mathrm{L}}$ and $\mathrm{C}_{\mathrm{M}}$ cycles other than making the hysteresis cycle wider by increasing the difference between the upstroke and downstroke.

When $\alpha_{\text {mean }}$ increased from $0^{\circ}$ to $10^{\circ}$, the $\mathrm{C}_{\mathrm{L}}$ and $\mathrm{C}_{\mathrm{M}}$ curves are altered significantly. The $\mathrm{C}_{\mathrm{L}}$ values are approximately double their static counterparts for high $\alpha$ due to dynamic stall occurrence. There is a presence of multiple sudden peaks in the $\mathrm{C}_{\mathrm{L}}$ data indicating the formation of multiple LEV. After the LEV, the airfoil is stalled until 
reattachment occurs when $\alpha$ is much lower than the static stall point. There is also a sudden peak in $\mathrm{C}_{\mathrm{M}}$ because as the vortex sweeps over the airfoil, it produces a rapid aft movement of the center of pressure from the LE towards the TE resulting in a large nose-down pitching moment on the airfoil. The TEF was able to decrease the magnitude of negative damping and in some cases eliminate it as was seen in the $\mathrm{C}_{\mathrm{M}}-\alpha$ plots. When $k$ increased from 0.06 to 0.1 a few significant differences arise: the $\mathrm{C}_{\mathrm{L}}$ peak increased indicating a larger and stronger LEV; there is a delay in the formation of LEV and flow reattachment; the difference between the upstroke and downstroke is larger, slightly widening the hysteresis loop; and the negative damping loops are longer and larger in magnitude.

$\Delta \mathrm{C}_{\mathrm{p}}$ contours for the different cases show a clearer picture of where the high suction peaks occur, or the formation of the primary and secondary LEV and how they move along the airfoil, or flow separation on the airfoil. $\operatorname{The} S T D\left(\Delta \mathrm{C}_{\mathrm{p}}\right)$ contour plots show the location of the boundary layer transition points and stall area. From the contour plots the patterns in $\mathrm{C}_{\mathrm{L}}$ and $\mathrm{C}_{\mathrm{M}}$ could be explained.

All the plots show how a TEF can influence $\mathrm{C}_{\mathrm{L}}, \mathrm{C}_{\mathrm{M}}, \mathrm{C}_{\mathrm{p}}, \mathrm{LEV}$, and stall. If the aim is to reduce $\mathrm{C}_{\mathrm{L}}$ fluctuations around the mean angle then it would be best to set $\phi=\pi / 2$. On the other hand, if the aim is to reduce $\mathrm{C}_{\mathrm{L}}$ fluctuations at the min and max then it would be best to set $\phi=0 \pi$. Now focusing on the $\mathrm{C}_{\mathrm{M}}$ plots, it was found $\phi=0 \pi$ decreases the peaks and reduces the negative damping loop in size. For $\phi=\pi / 2$ it was found that the entire $\mathrm{C}_{\mathrm{M}}$ loop causes negative damping promoting stall flutter under some structural conditions. It is recommended then to use $\phi=0 \pi$ because it reduces the min/max of the $\mathrm{C}_{\mathrm{L}}$ and $\mathrm{C}_{\mathrm{M}}$ cycle and reduces negative damping.

\section{Funding Sources}

We acknowledge the support of the Natural Sciences and Engineering Research Council of Canada (NSERC) through a grant to the second author.

\section{References}

[1] Shipley, D. E., Miller, M. S., and Michael C. Robinson, "Dynamic Stall Occurrence on a Horizontal Axis Wind Turbine Blade," Fourteenth ASME-ETCE Wind Energy Symposium, Vol. 14, No. July, 1995.

[2] Barlas, T. K., and van Kuik, G. A. M., "Review of state of the art in smart rotor control research for wind turbines," Progress in Aerospace Sciences, Vol. 46, No. 1, 2010, pp. 1-27. https://doi.org/https://doi.org/10.1016/j.paerosci.2009.08.002

[3] W. Carr, L., W. McAlister, K., and J. McCroskey, W., “Analysis of the Development of Dynamic Stall Based on Oscillating Airfoil Experiments," Tech. rep., 1977.

[4] McCroskey, W. J., "Unsteady Airfoils," Annual Review of Fluid Mechanics, Vol. 14:285-311, 1982, pp. $285-311$. https://doi.org/https://doi.org/10.1146/annurev.fl.14.010182.001441

[5] Corke, T. C., and Thomas, F. O., "Dynamic Stall in Pitching Airfoils: Aerodynamic Damping and Compressibility Effects," 
Annual Review of Fluid Mechanics, Vol. 47, No. 1, 2015, pp. 479-505. https://doi.org/https://doi.org/10.1146/annurev-fluid010814-013632

[6] Leishman, J. G., "Dynamic stall experiments on the NACA 23012 aerofoil," Experiments in Fluids, Vol. 9, No. 1-2, 1990, pp. 49-58. https://doi.org/https://doi.org/10.1007/BF00575335

[7] Gharali, K., and Johnson, D. A., "Dynamic stall simulation of a pitching airfoil under unsteady freestream velocity," Journal of Fluids and Structures, Vol. 42, 2013, pp. 228-244. https://doi.org/https://doi.org/10.1016/j.jfluidstructs.2013.05.005.

[8] Gharali, K., and Johnson, D. A., "PIV-based load investigation in dynamic stall for different reduced frequencies," Experiments in Fluids, Vol. 55, No. 8, 2014. https://doi.org/https://doi.org/10.1007/s00348-014-1803-8

[9] Gharali, K., and Johnson, D. A., "Effects of nonuniform incident velocity on a dynamic wind turbine airfoil," Wind Energy, Vol. 18, No. 2, 2015, pp. 237-251. https://doi.org/https://doi.org/10.1002/we.1694.

[10] Lee, T., and Gerontakos, P., "Investigation of flow over an oscillating airfoil," Journal of Fluid Mechanics, Vol. 512, 2004, pp. 313-341. https://doi.org/https://doi.org/10.1017/S0022112004009851.

[11] Wang, S., Ingham, D. B., Ma, L., Pourkashanian, M., and Tao, Z., "Numerical investigations on dynamic stall of low Reynolds number flow around oscillating airfoils," Computers and Fluids, Vol. 39, No. 9, 2010, pp. 1529-1541. https://doi.org/https: //doi.org/10.1016/j.compfluid.2010.05.004

[12] Baik, Y. S., Bernal, L. P., Granlund, K., and Ol, M. V., "Unsteady force generation and vortex dynamics of pitching and plunging aerofoils," Journal of Fluid Mechanics, Vol. 709, 2012, pp. 37-68. https://doi.org/https://doi.org/10.1017/jfm.2012.318.

[13] Pitt Ford, C. W., and Babinsky, H., "Lift and the leading-edge vortex," Journal of Fluid Mechanics, Vol. 720, 2013 , pp. $280-313$. https://doi.org/https://doi.org/10.1017/jfm.2013.28

[14] Cebeci, T., Platzer, M., Chen, H., Chang, K.-C., and Shao, J. P., Analysis of Low-Speed Unsteady Airfoil Flows, Springer New York, 2005.

[15] Masdari, M., Seyednia, M., and Tabrizian, A., "An experimental loading study of a pitching wind turbine airfoil in near- and post-stall regions," Journal of Mechanical Science and Technology, Vol. 32, No. 8, 2018, pp. 3699-3706. https://doi.org/https://doi.org/10.1007/s12206-018-0722-7

[16] Leishman, J. G., Principles of Helicopter Aerodynamics, $2^{\text {nd }}$ ed., Cambridge University Press, 2006.

[17] Green, R., Gillies, E., and Wang, Y., “Trailing edge flap flow control for dynamic stall,” Aeronautical Journal, Vol. 115, No. 1170, 2011, pp. 493-503. https://doi.org/https://doi.org/10.1017/S0001924000006138

[18] Davis, G. L., Feszty, D., and Nitzsche, F., "Trailing Edge Flow Control for the Mitigation of Dynamic Stall Effects," 31 st European Rotorcraft Forum, 2005, pp. 1-11. 
[19] McCroskey, W., Carr, L., and McAlister, K., "Dynamic Stall Experiments on Oscillating Airfoils," AIAA Journal, Vol. 14, No. 1, 1976, pp. 57-63. https://doi.org/https://doi.org/10.2514/3.61332

[20] Boutilier, M. S., and Yarusevych, S., "Parametric study of separation and transition characteristics over an airfoil at low Reynolds numbers," Experiments in Fluids, Vol. 52, No. 6, 2012, pp. 1491-1506. https://doi.org/https://doi.org/10.1007/s00348-012$1270-\mathrm{Z}$

[21] Raiola, M., Discetti, S., Ianiro, A., Samara, F., Avallone, F., and Ragni, D., "Smart Rotors: Dynamic-Stall Load Control by Means of an Actuated Flap,” AIAA Journal, Vol. 56, No. 4, 2018, pp. 1-14. https://doi.org/https://doi.org/10.2514/1.J056342.

[22] Sun, M., and Sheikh, S., "Dynamic stall suppression on an oscillating airfoil by steady and unsteady tangential blowing," Aerospace Science and Technology, Vol. 3, No. 6, 1999, pp. 355-366. https://doi.org/https://doi.org/10.1016/S1270-9638(00) 86426-3

[23] Weaver, D., McAlister, K., and Tso, J., “Control of VR-7 Dynamic Stall by Strong Steady Blowing," Journal of Aircraft, Vol. 41, No. 6, 2004, pp. 1404-1413. https://doi.org/https://doi.org/10.2514/1.4413

[24] Smith, B. L., and Glezer, A., "The formation and evolution of synthetic jets," Physics of Fluids, Vol. 10, No. 9, 1998, pp. 2281-2297. https://doi.org/https://doi.org/10.1063/1.869828

[25] Corke, T. C., Bowles, P. O., He, C., and Matlis, E. H., "Sensing and control of flow separation using plasma actuators," Philosophical Transactions of the Royal Society A: Mathematical, Physical and Engineering Sciences, Vol. 369, No. 1940, 2011, pp. 1459-1475. https://doi.org/https://doi.org/10.1098/rsta.2010.0356.

[26] Post, M. L., and Corke, T. C., "Separation Control Using Plasma Actuators: Dynamic Stall Vortex Control on Oscillating Airfoil,” AIAA Journal, Vol. 44, No. 12, 2006, pp. 3125-3135. https://doi.org/https://doi.org/10.2514/1.22716

[27] Heine, B., Mulleners, K., Joubert, G., and Raffel, M., "Dynamic Stall Control by Passive Disturbance Generators," AIAA Journal, Vol. 51, No. 9, 2013, pp. 2086-2097. https://doi.org/https://doi.org/10.2514/1.J051525.

[28] Martin, P., Wilson, J., Berry, J., Wong, T.-C., Moulton, M., and McVeigh, M., "Passive Control of Compressible Dynamic Stall," 26th AIAA Applied Aerodynamics Conference, American Institute of Aeronautics and Astronautics, 2008. https://doi.org/https: //doi.org/10.2514/6.2008-7506

[29] Rennie, R. M., and Jumper, E. J., "Experimental measurements of dynamic control surface effectiveness," Journal of Aircraft, Vol. 33, No. 5, 1996, pp. 880-887. https://doi.org/https://doi.org/10.2514/3.47030

[30] Vipperman, J. S., Clark, R. L., Conner, M., and Dowell, E. H., "Experimental Active Control of a Typical Section Using a Trailing-Edge Flap,” Journal of Aircraft, Vol. 35, No. 2, 1998, pp. 224-229. https://doi.org/https://doi.org/10.2514/2.2312

[31] Nguyen, K., “Active Control of Helicopter Blade Stall,” Journal of Aircraft, Vol. 35, No. 1, 1998, pp. 91-98. https://doi.org/https: //doi.org/10.2514/2.2264 
[32] Feszty, D., Gillies, E. A., and Vezza, M., “Alleviation of Airfoil Dynamic Stall Moments via Trailing-Edge Flap Flow Control,” AIAA Journal, Vol. 42, No. 1, 2004, pp. 17-25. https://doi.org/https://doi.org/10.2514/1.853

[33] Lee, T., and Gerontakos, P., "Dynamic Stall Flow Control via a Trailing-Edge Flap," AIAA Journal, Vol. 44, No. 3, 2006, pp. 469-480. https://doi.org/https://doi.org/10.2514/1.17263.

[34] Gerontakos, P., and Lee, T., “Trailing-Edge Flap Control of Dynamic Pitching Moment,” AIAA Journal, Vol. 45, No. 7, 2007, pp. 1688-1694. https://doi.org/https://doi.org/10.2514/1.27577

[35] Gerontakos, P., and Lee, T., "PIV study of flow around unsteady airfoil with dynamic trailing-edge flap deflection," Experiments in Fluids, Vol. 45, No. 6, 2008, pp. 955-972. https://doi.org/https://doi.org/10.1007/s00348-008-0514-4

[36] Lee, T., and Su, Y. Y., "Unsteady airfoil with a harmonically deflected trailing-edge flap," Journal of Fluids and Structures, Vol. 27, No. 8, 2011, pp. 1411-1424. https://doi.org/https://doi.org/10.1016/j.jfluidstructs.2011.06.008

[37] Somers, D. M., “The S833 , S834 , and S835 Airfoils,” Tech. rep., NREL/SR-500-36340, 2005. https://doi.org/10.2172/15020040

[38] Kurelek, J. W., Lambert, A. R., and Yarusevych, S., "Coherent Structures in the Transition Process of a Laminar Separation Bubble,” AIAA Journal, Vol. 54, No. 8, 2016, pp. 2295-2309. https://doi.org/https://doi.org/10.2514/1.J054820

[39] Holman, J., Experimental Methods for Engineers, seventh ed., McGraw-Hill Publishing Company, 2001.

[40] Samara, F., and Johnson, D. A., "In-blade Load Sensing on 3D Printed Wind Turbine Blades Using Trailing Edge Flaps," Journal of Physics: Conference Series, Vol. 1037, 2018, p. 052023. https://doi.org/10.1088/1742-6596/1037/5/052023

[41] Morote, J., "Angle of attack distribution on wind turbines in yawed flow," Wind Energy, Vol. 19, No. 4, 2016 , pp. 681-702. https://doi.org/https://doi.org/10.1002/we.1859

[42] Burton, T., Jenkins, N., Sharpe, D., and Bossanyi, E., Wind Energy Handbook, $2^{\text {nd }}$ ed., Wiley, 2011.

[43] Gallant, T., and Johnson, D. A., "Flow angle measurement of a yawed turbine and comparison to models," Wind Energy Science Discussions, Vol. 2, No. January, 2017, pp. 1-17. https://doi.org/https://doi.org/10.5194/wes-2016-57

[44] Moscardi, A., and Johnson, D. A., "A compact in-blade five hole pressure probe for local inflow study on a horizontal axis wind turbine," Wind Engineering, Vol. 40, No. 4, 2016, pp. 360-378. https://doi.org/https://doi.org/10.1177/0309524X16650766

[45] Barlow, J. B., Rae, W. H., and Pope, A., Low-Speed Wind Tunnel Testing, John Wiley \& Sons Ltd, 1999.

[46] Ramsay, R., Hoffman, M., and Gregorek, G., "Effects of grit roughness and pitch oscillations on the S809 airfoil," Tech. Rep. December, National Renewable Energy Laboratory (NREL), Golden, CO, dec 1995. https://doi.org/10.2172/205563

[47] Drela, M., and Youngren, H., "XFOIL 6.97,", 2000. https://doi.org/3, URL https://web.mit.edu/drela/Public/web/xfoil/.

[48] Larsen, J. W., Nielsen, S. R., and Krenk, S., "Dynamic stall model for wind turbine airfoils," Journal of Fluids and Structures, Vol. 23, No. 7, 2007, pp. 959-982. https://doi.org/https://doi.org/10.1016/j.jfluidstructs.2007.02.005 
[49] Bak, C., Gaunaa, M., Andersen, P. B., Buhl, T., Hansen, P., and Clemmensen, K., "Wind Tunnel Test on Wind Turbine Airfoil with Adaptive Trailing Edge Geometry," 45th AIAA Aerospace Sciences Meeting and Exhibit, 2007, pp. 1-12. https://doi.org/https://doi.org/10.2514/6.2007-1016 